\title{
Wave fluxes of equatorial Kelvin waves and QBO zonal wind forcing derived from SABER and ECMWF temperature space-time spectra
}

\author{
M. Ern and P. Preusse \\ Institute of Chemistry and Dynamics of the Geosphere (ICG-1), Forschungszentrum Jülich, Jülich, Germany \\ Received: 19 January 2009 - Published in Atmos. Chem. Phys. Discuss.: 3 March 2009 \\ Revised: 28 May 2009 - Accepted: 6 June 2009 - Published: 17 June 2009
}

\begin{abstract}
The quasi-biennial oscillation (QBO) of the zonal mean zonal wind is a dynamical phenomenon of the tropical middle atmosphere. Influences of the QBO can even be found at mid and high latitudes. It is widely accepted that the phase descent of alternating tropical easterlies and westerlies is driven by atmospheric waves of both global scale (equatorial wave modes like Kelvin, equatorial Rossby, Rossbygravity, or inertia-gravity waves), as well as mesoscale gravity waves. However, the relative distribution of the different types of waves to the forcing of the QBO winds is highly uncertain. This is the case because until recently there were no high resolution long-term global measurements in the stratosphere. In our study we estimate Kelvin wave momentum flux and the contribution of zonal wind forcing by Kelvin waves based on space-time spectra determined from both Sounding of the Atmosphere using Broadband Emission Radiometry (SABER) temperature measurements as well as temperatures from European Centre for Medium-Range Weather Forecasts (ECMWF) operational analyses. Peak values of total Kelvin wave zonal wind forcing found are about $0.2 \mathrm{~m} / \mathrm{s} /$ day. There is good agreement between SABER and ECMWF results. Altitude-time cross sections are shown and the results are compared to the total wave forcing required to balance the background atmosphere. Sometimes Kelvin wave forcing is sufficient to explain almost the whole total wave forcing required for the momentum balance during the transition from QBO easterly to westerly winds. This is especially the case during the periods of strong westerly wind shear when the zonal wind is between -20 and $10 \mathrm{~m} / \mathrm{s}$ at the equator in the altitude range 20 to $35 \mathrm{~km}$. During other parts of the phases of strong westerly wind shear, however, the contribution of Kelvin waves can be comparably low and the missing wave forcing, which is often attributed
\end{abstract}

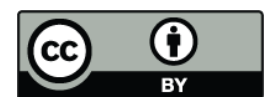

Correspondence to: M. Ern (m.ern@fz-juelich.de) to mesoscale gravity waves or intermediate scale waves, can be the by far dominant contribution of the QBO forcing. It is also found that seasonal variations of Kelvin wave accelerations could play an important role for the maintenance of the QBO westerly wind jets in the lower stratosphere.

\section{Introduction}

The tropics are an important region for the dynamics of the whole atmosphere. Characteristic for the tropical region are enhanced solar heating and deep convective processes. The residual mean circulation is directed upward in the tropics. As a consequence of the deep convective processes and the mean upwelling tropospheric constituents can more easily enter the stratosphere than in the mid and high latitudes. Deep convection and the latent heat release in the tropics are also the source processes of a broad spectrum of atmospheric waves of both global scale (Rossby-gravity, equatorial Rossby, inertia-gravity and Kelvin waves) as well as mesoscale gravity waves (e.g., Salby and Garcia, 1987; Bergman and Salby, 1994; Ricciardulli and Garcia, 2000).

One of the most important phenomena of the dynamics in the tropics is the quasi-biennial oscillation (QBO): The zonal mean zonal wind alternates between easterlies and westerlies with a period of about 28 months. The region of QBO winds is confined to the latitude band between about $15^{\circ} \mathrm{S}$ and $15^{\circ} \mathrm{N}$ and is observed mainly at stratospheric altitudes between about 18 and $40 \mathrm{~km}$. At higher altitudes in the upper stratosphere and large parts of the mesosphere the situation is different. In this altitude region the zonal mean zonal wind is dominated by a semi-annual oscillation (SAO). The wind changes between strong easterlies and strong westerlies with a dominant period of 6 months. In the upper mesosphere the zonal mean zonal wind is again dominated by the QBO (Baldwin et al., 2001).

Published by Copernicus Publications on behalf of the European Geosciences Union. 
It has been shown by Lindzen and Holton (1968) that the QBO is a wave driven circulation with waves of eastward as well as westward phase velocity playing an important role. After the theory for global scale wave modes developed by Matsuno (1966) had been confirmed by the observations of Wallace and Kousky (1968) for equatorial Kelvin waves and Yanai and Maruyama (1966) for Rossby-gravity waves, in a later work Holton and Lindzen (1972) demonstrated that the forcing by those global scale equatorial waves modes would be sufficient for driving the QBO and could fully explain the observed descent of easterly and westerly wind phases.

However, with the discovery of the so called "tape recorder effect" by Mote et al. $(1995,1996)$ it was possible to quantify the residual mean upward velocity from seasonal variations of water vapor observations in the tropical stratosphere. The observed residual mean upwelling in the lower stratosphere is of the order of about $0.1-0.6 \mathrm{~mm} / \mathrm{s}$ (Rosenlof, 1995; Dunkerton, 1997; Schoeberl et al., 2008). This effect is too large to be neglected, and Dunkerton (1997) concluded that additional wave forcing by mesoscale gravity waves is required to compensate the vertical advection due to the residual mean upwelling so that the observed descent of QBO wind phases can still be explained. This additional wave forcing was estimated to be about 2-4 times as strong as the forcing due to global scale equatorial wave modes (Dunkerton, 1997).

There are several studies that try to attribute the forcing of the QBO in the stratosphere or the SAO in the upper stratosphere and in the mesosphere to the different types of global scale waves (e.g., Hitchman and Leovy, 1988; Sato and Dunkerton, 1997; Dunkerton, 1997; Canziani and Holton, 1998). Except for Sato and Dunkerton (1997) these studies are all based on waves having zonal wavenumbers 1 and 2 or 1-3 only. While the estimates of Sato and Dunkerton (1997) and Dunkerton (1997) are based on local data sets from single measurement stations, the analyses of Hitchman and Leovy (1988) and Canziani and Holton (1998) are based on global satellite data, but do not cover a full QBO cycle.

It has been shown by Tindall et al. (2006a,b) that it is not sufficient to consider zonal wavenumbers $1-3$ because also higher zonal wavenumbers contribute significantly to the vertical flux of zonal wave momentum. And from results of single station measurements the global average contribution of the different wave types cannot be derived. Therefore there is still large uncertainty about how the different types of global scale waves contribute to the forcing of the QBO and SAO. The deficiencies of previous studies are mainly due to the fact that up to now there were no long-term high spatial resolution (both horizontally and vertically) global observations of the stratosphere.

However, for a more complete understanding of the QBO and its mechanisms the knowledge of the different forcing terms would be required. In particular, because effects due to the QBO do not only play an important role in the tropics, but also in other parts of the atmosphere. Many processes in atmospheric chemistry and dynamics in the stratosphere and mesosphere (even at high latitudes) are modulated or influenced by the QBO, showing the importance of the QBO and of tropical wave activity (Baldwin et al., 2001).

Some processes wave activity in the tropics and the QBO are important for are the mean upwelling observed in the equatorial region (e.g., Semeniuk and Shepherd, 2001) as well as mixing processes both vertically (e.g., Fujiwara et al., 1998; Fujiwara and Takahashi, 2001) and across the subtropical mixing barrier, which is more stable during QBO easterly phases (e.g., Shuckburgh et al., 2001). In addition, Kelvin waves play an important role for the dehydration of the tropical tropopause region (e.g., Fujiwara et al., 2001; Zhou and Holton, 2002; Hatsushika and Yamazaki, 2003; Eguchi and Shiotani, 2004; Jensen and Pfister, 2004; Immler et al., 2008). Even processes at high latitudes are modulated by the QBO. For example, the polar vortices are influenced by the QBO (Angell and Korshover, 1975; Baldwin and Dunkerton, 1998), and there are also effects on the weather in the troposphere (Baldwin and Dunkerton, 1999).

Because the driving of the QBO is a very sensitive result of wave mean flow interaction with very different types of waves contributing, the simulation of the QBO in general circulation models (GCMs) is very difficult and there are only few realistic simulations of the QBO (e.g., Takahashi, 1999; Scaife et al., 2000; Giorgetta et al., 2002, 2006).

All these examples show that it is very important to better quantify the contributions of the different types of waves to the QBO forcing in the tropics. In addition, recent high resolution GCMs have started to resolve part of the spectrum of mesoscale gravity waves (e.g., Hamilton, 2006; Hamilton et al., 2008; Watanabe et al., 2008; Kawatani et al., 2009). Therefore experimental constraints for the different kinds of wave forcing are severely needed to validate these model results.

The situation of the observational data now has changed with the data sets of the new satellite instruments Sounding of the Atmosphere using Broadband Emission Radiometry (SABER) and High Resolution Dynamics Limb Sounder (HIRDLS), as well as six GPS receivers forming the Constellation Observing System for Meteorology, Ionosphere and Climate (COSMIC). All these high resolution data sets are now longer than about 2.5 years, and measurements are still ongoing for SABER and COSMIC. First results for global scale equatorial wave modes have already been shown, for example, by Garcia et al. (2005); Ern et al. $(2008,2009)$ or Alexander et al. (2008). These new sensors provide the high resolution data sets required for a better assessment of the different wave contributions to the total QBO forcing. Although the spatial resolution still is not good enough to directly estimate the forcing by mesoscale gravity waves with horizontal wavelengths in the range of about 100 up to several thousand $\mathrm{km}$, the contribution of the global scale equatorial wave modes can now be estimated for at least one QBO period. 
In our work we will quantify the contribution of equatorial Kelvin waves to the forcing of the QBO. Kelvin waves are the most prominent global scale equatorially trapped wave mode in atmospheric temperatures (e.g., Tindall et al., 2006a). They are symmetric with respect to the equator, trapped at latitudes between about $20^{\circ} \mathrm{S}$ and $20^{\circ} \mathrm{N}$ in the stratosphere and travel eastward.

Because Kelvin waves interact with the QBO winds Kelvin wave activity itself is modulated by the QBO. Enhanced Kelvin wave activity is observed during QBO westward phases when the propagation conditions are favorable for eastward traveling waves. Kelvin waves dissipate when they approach the zonal wind reversal from westward to eastward wind. Accordingly, they transfer momentum to the background wind and thereby contribute to the reversal of the zonal wind direction (e.g. Holton and Lindzen, 1972; Campbell and Shepherd, 2005a,b).

To quantify the effect of Kelvin waves for driving the QBO we have to determine the wave forcing, i.e. the vertical gradient of the vertical flux of horizontal momentum $\overline{u^{\prime} w^{\prime}}$, with $u^{\prime}$ and $w^{\prime}$ the horizontal and vertical wind perturbations of the zonal mean state due to Kelvin waves. The overbar denotes temporal averaging (see also Appendix A). Our analysis will however be based on zonal wavenumber/frequency space-time spectra calculated from SABER as well as European Centre for Medium-Range Weather Forecasts (ECMWF) temperatures, and not horizontal and vertical wind speeds. Measurement techniques are by far not precise enough to measure vertical wind perturbations of the order of $1 \mathrm{~cm} / \mathrm{s}$ globally from satellite. Therefore the polarization relations for Kelvin waves have to be used to determine momentum flux spectra from the temperature spacetime spectra.

In Sect. 2 we will first describe the space-time spectral analysis, how Kelvin waves force the zonal mean wind, and how we transform the temperature space-time spectra into momentum flux spectra. In Sect. 3 we will discuss the basic properties of average SABER and ECMWF temperature and momentum flux spectra as well as the spatial and temporal variation of Kelvin wave momentum flux and compare the results with estimates of other authors. In Sect. 4 we will also discuss the spatial and temporal variation of the zonally averaged forcing of the zonal mean flow by Kelvin waves and compare the results with previous findings. An error discussion will be given in Sect. 5. The results are summarized in Sect. 6.

\section{Kelvin wave spectra and zonal wind forcing}

\subsection{Theory of equatorial wave modes}

A theoretical description of planetary scale equatorial wave modes was first given by Matsuno (1966) who derived the properties of the different wave types from solutions of the shallow-water model on an equatorial beta plane, resulting in the following dispersion relation:

$\frac{\sqrt{g h_{e}}}{\beta}\left(\frac{\hat{\omega}^{2}}{g h_{e}}-k^{2}-\frac{k \beta}{\hat{\omega}}\right)=2 n+1, \quad n=0,1,2, \ldots$

with $\hat{\omega}$ the intrinsic frequency of the wave, $k$ the zonal wavenumber, $\beta$ the meridional gradient of the Coriolis parameter, $g$ the gravity acceleration, $n$ the order of the solution, and $h_{e}$ the so-called equivalent depth. The wave modes described by Eq. (1) are trapped near the equator. For a more detailed discussion of the different wave modes see, for example, Matsuno (1966).

The equivalent depth is connected with the vertical wavenumber $m$ as given in Eq. (2) (e.g., Wu et al., 2000; Lindzen, 2003):

$m^{2}=\left(\frac{N^{2}}{g h_{e}}-\frac{1}{4 H^{2}}\right)$

with $N$ the buoyancy frequency, and $H$ the pressure scale height.

For the special case of Kelvin waves, which is usually labeled $n=-1$ and is not covered by Eq. (1) (see Matsuno, 1966), the dispersion relation is reduced to:

$\hat{\omega}=-\sqrt{g h_{e}} k \approx-N k / m$

From Eq. (3) we see that the phase speed $\hat{\omega} / k$ of a Kelvin wave is directly coupled with the equivalent depth. Under the assumption of zero background wind equivalent depths of 8,90 and $2000 \mathrm{~m}$ correspond to intrinsic phase speeds of about 9,30 , and $140 \mathrm{~m} / \mathrm{s}$ (see Eq. 3) and vertical wavelengths of about 3,9 and $50 \mathrm{~km}$ in the stratosphere, or about 6,19 and $100 \mathrm{~km}$ in the troposphere due to the lower buoyancy frequency $N$ there (see Eq. 2).

It should be noted that the frequencies $\omega$ that are observed by satellite instruments and most other observing systems are ground based (Eulerian) frequencies. These frequencies remain unchanged in cases of non-zero background wind and spectral features in ground based frequency/zonal wavenumber spectra like in our paper are not Doppler shifted. On the other hand intrinsic wave frequencies $\hat{\omega}$ will be Doppler shifted in case of non-zero background wind according to:

$\hat{\omega}=\omega-k \bar{u}$

with $k$ the horizontal wavenumber and $\bar{u}$ the background wind. Also, according to the dispersion relation (Eq. 3), the vertical wavelength changes when $\hat{\omega}$ is Doppler shifted. A more detailed discussion can, for example, be found in Ern et al. (2008).

\subsection{Space-time spectral analysis}

The spectral signatures of the different global scale equatorial wave modes can be identified easily in zonal wavenumber/frequency spectra because of their characteristic wave bands (see e.g., Wheeler and Kiladis, 1999; Ern et al., 2008). 
In our study we will derive zonal wavenumber/frequency spectra from temperatures measured by the Sounding of the Atmosphere using Broadband Emission Radiometry (SABER) instrument onboard the Thermosphere Ionosphere Mesosphere Energetics and Dynamics (TIMED) satellite, as well as from temperatures of European Centre for MediumRange Weather Forecasts (ECMWF) operational analyses.

To derive space-time spectra we follow the method described in Ern et al. (2008). The data are arranged into latitude/altitude bins. For the so obtained longitude/time-series for each of the bins a two-dimensional Fourier transform can then be carried out, resulting in zonal wavenumber/frequency spectra. In order to investigate temporal variations of the space-time spectra, we subdivide the whole data set into nonoverlapping time windows and carry out a windowed spacetime Fourier analysis instead of analyzing the data set as a whole. A time window length of 31 days is used, which accounts for the seasonal variations expected in the data analyzed, and at the same time provides good spectral resolution of the space-time spectra.

Different from Ern et al. (2008), we do not calculate symmetric and antisymmetric spectra with respect to the equator for a given altitude. Instead, like in Ern et al. (2009), the spectral analyses for each latitude and altitude are used without meridional averaging. The first reason for doing so is because Kelvin waves are the by far dominant wave mode in temperature. Therefore the Kelvin wave signal will be almost not distorted by other wave modes. The second reason is that by calculating symmetric and antisymmetric spectra automatically symmetry or anti-symmetry of the different wave modes with respect to the equator is assumed and latitudinal averaging is introduced by combining northern and southern latitudes. In our work we want to check upon this assumption and will calculate latitudinally resolved results for southern and northern latitudes independently.

The SABER instrument measures temperatures and several trace gases from the tropopause region to above $100 \mathrm{~km}$ (e.g., Mlynczak, 1997; Russell et al., 1999; Yee et al., 2003). Like in Ern et al. (2008) we use version 1.06 temperature data, and the space-time spectra are calculated from residual temperatures in non-overlapping latitude bins from $20^{\circ} \mathrm{S}$ to $20^{\circ} \mathrm{N}$ in 4 deg steps. The width of the latitude bins is chosen according to the horizontal sampling distance of about $500 \mathrm{~km}$ along the satellite track. Due to the orbit geometry of the TIMED satellite zonal wavenumbers up to 6-7 and frequencies up to 1 cycle/day can be resolved by the asynoptic sampling. The SABER data used are given on fixed geometric altitudes from $15 \mathrm{~km}$ to above $100 \mathrm{~km}$ in $1-\mathrm{km}$ steps. This is somewhat better than the vertical resolution given by the instantaneous vertical field-of-view of about $2 \mathrm{~km}$.

Since satellite data are sampled asynoptically, i.e., are not given on a regular longitude/time grid, simple twodimensional Fast Fourier Transform (FFT) cannot be applied. For a more detailed discussion see Salby (1982a,b). In our approach we use a least-squares method, similar to the one by Wu et al. (1995), to derive amplitudes and phases of the independent space-time Fourier coefficients for each of the latitude/altitude bins.

Like in Ern et al. (2008) we also calculate space-time spectra from temperatures of ECMWF operational analyses. The ECMWF model used for operational analyses consists of a high resolution deterministic model to simulate atmospheric dynamics. Starting from 21 November 2000 the longitudinal resolution was T511 (about $40 \mathrm{~km}$ at the equator). In total 60 vertical levels were used from the ground to the model top at $0.1 \mathrm{hPa}$. On 1 February 2006 the horizontal model resolution was further upgraded to T799, the model top was raised to $0.01 \mathrm{hPa}$, and 91 vertical levels were used. Further information can, for example, also be found in Jung and Leutbecher (2007). The history of ECMWF model versions can be found on the ECMWF web pages (see ECMWF, 2009).

Numerous experimental data sets are assimilated into ECMWF. Starting from 12 September 2000 the data assimilation is based on a $12 \mathrm{~h} 4 \mathrm{~d}-$ Var system. The evolution of the ECMWF observing system until 2002 is, for example, described in Uppala et al. (2005). The observing system is based on conventional data sources as well as satellite observations. Conventional data sources are, for example, wind profiler data, radiosonde temperatures and humidity, weather data from aircraft, or data from land surface weather stations, ships and buoys. Satellite observations entering the ECMWF data assimilation system are mainly radiances measured by geostationary satellites, as well as satellites in low Earth orbit. For the data assimilation in the stratosphere especially the observations from satellites in low Earth orbit are very important (for example, TOVS/ATOVS radiances).

Of course, it could be possible that results obtained from ECMWF data might change as a consequence of model revisions or because additional experimental data sets are included. However, there are no indications for such effects in our results presented.

For our spectral analysis the ECMWF temperatures are gridded down to 9 deg resolution in longitude from originally $1 \mathrm{deg}$, resulting in a maximum zonal wavenumber of 20 that can be resolved. The original resolution of $1 \mathrm{deg}$ meridionally has been retained unchanged and space-time spectra are calculated for each latitude in 1 deg steps. Frequencies up to 2 cycles/day can be resolved since the ECMWF analyses are available every $6 \mathrm{~h}$ at 00:00, 06:00, 12:00, and 18:00 GMT. The ECMWF data set is given on a set of pressure levels which we convert to pressure altitudes using a constant scale height of $7 \mathrm{~km}$. For the ECMWF data, which are given on a regular grid, zonal wavenumber/frequency spectra can be calculated easily by applying a two-dimensional FFT.

Usually, momentum fluxes due to atmospheric waves are calculated from wind perturbations of the zonal averages. Different from this, in our study we will rely on temperature spectra because high precision long-term satellite data sets (like the SABER data) are mainly based on temperatures. Stratospheric wind data from satellites are still not precise 
enough for this kind of wave analysis. Measurements of the vertical wind would have to be far better than about $1 \mathrm{~cm} / \mathrm{s}$. In addition, the ECMWF temperature spectra were directly validated with measured SABER temperature spectra by Ern et al. (2008). The quality of ECMWF wind spectra has not been validated by measurements so far. In addition, in temperature spectra the Kelvin wave signal will be the by far most prominent spectral component and less contaminated by other waves than in wind spectra (see also Tindall et al., 2006a,b).

It should also be kept in mind that, by using a windowed Fourier analysis for generating zonal wavenumber/frequency spectra, all our results will represent zonal averages additionally averaged over the 31-day time windows.

\subsection{Zonal wind forcing by Kelvin waves}

As mentioned above, we will investigate the zonal mean Kelvin wave momentum fluxes and zonal wind forcing additionally averaged over the 31-day time windows of our spectral analysis. The zonal mean momentum balance is given by:

$\frac{\mathrm{d} \bar{u}}{\mathrm{dt}}-f \bar{v}=-F_{\text {wave }}$

with $(\bar{u}, \bar{v}, \bar{w})$ the zonal mean background wind vector, $f$ the Coriolis frequency, and $F_{\text {wave }}$ the total acceleration contributed by atmospheric waves (e.g., Andrews and McIntyre, 1976).

In our work zonal mean temperatures $T$ as well as the zonal mean background wind vector will be taken from ECMWF, averaged not only over the longitudes, but also over the 31-day time windows of our analysis.

Following Eq. (3.1a) in Andrews and McIntyre (1976) Eq. (5) can be written as follows:

$$
\begin{aligned}
\frac{\partial \bar{u}}{\partial t}+\bar{v}\left(\frac{\partial \bar{u}}{\partial y}-f\right)+\bar{w} \frac{\partial \bar{u}}{\partial z} & =-\frac{\partial}{\partial y}\left(\overline{u^{\prime} v^{\prime}}\right)-\frac{\partial}{\partial z}\left(\overline{u^{\prime} w^{\prime}}\right)-\bar{X} \\
& =-F_{\text {wave }}
\end{aligned}
$$

with $\left(u^{\prime}, v^{\prime}, w^{\prime}\right)$ the vector of wind perturbations with respect to the zonal average, $y$ the meridional coordinate, $z$ the vertical coordinate, and $f$ the Coriolis frequency. The total wave forcing is split up into three terms with: $\overline{u^{\prime} v^{\prime}}$ the meridional flux of zonal wave momentum and $\overline{u^{\prime} w^{\prime}}$ the vertical flux of zonal wave momentum, the overbar denotes temporal averaging. The term $\bar{X}$ is another unspecified forcing term which is introduced to capture further forcing not contained in the resolved wave perturbations of the background. For example, gravity wave drag parameterizations which are commonly used in GCMs would contribute to $\bar{X}$.

Our study is focused on Kelvin wave forcing alone. We therefore split up the total wave forcing term in the following way:

$F_{\text {wave }}=F_{\mathrm{KW}}+F_{\text {other }}$ with $F_{\mathrm{KW}}$ the forcing purely due to Kelvin waves (for this term it is assumed that no other waves are present), and $F_{\text {other }}$ the forcing with other waves involved (other global scale equatorial waves modes as well as gravity waves).

Since for Kelvin waves $v^{\prime}=0$, the forcing $F_{\mathrm{KW}}$ exerted by Kelvin waves is given by the vertical gradient of the flux of zonal momentum due to Kelvin waves alone $\left(\mathcal{F}_{p x}\right)$ divided by the atmospheric density $\bar{\varrho}_{0}$ at the reference altitude:

$F_{\mathrm{KW}}=\frac{1}{\bar{\varrho}_{0}} \frac{\partial \mathcal{F}_{p x}}{\partial z}=\frac{1}{\bar{\varrho}_{0}} \frac{\partial}{\partial z}\left(\bar{\varrho}_{0} \overline{u_{\mathrm{KW}}^{\prime} w_{\mathrm{KW}}^{\prime}}\right)=\frac{\partial}{\partial z}\left(\overline{u_{\mathrm{KW}}^{\prime} w_{\mathrm{KW}}^{\prime}}\right)$

with $u_{\mathrm{KW}}^{\prime}$ and $w_{\mathrm{KW}}^{\prime}$ the zonal and vertical wind perturbations due to Kelvin waves. In the following we will omit the subscript "KW", and perturbations of the zonal mean will be considered to be purely due to Kelvin waves.

The momentum balance Eq. (6) then becomes:

$$
\frac{\partial \bar{u}}{\partial t}+\bar{v}\left(\frac{\partial \bar{u}}{\partial y}-f\right)+\bar{w} \frac{\partial \bar{u}}{\partial z}=-F_{\text {other }}-\frac{1}{\bar{\varrho}_{0}} \frac{\partial \mathcal{F}_{p x}}{\partial z}
$$

This means to derive the wave forcing due to Kelvin waves we have to estimate the vertical gradient of Kelvin wave momentum flux $\partial \mathcal{F}_{p x} / \partial z$.

Strictly speaking the vertical flux of zonal momentum $\mathcal{F}_{p x}$ is given by $\mathcal{F}_{p x}=\bar{\varrho}_{0} \overline{u^{\prime} w^{\prime}}$ i.e., the term $\overline{u^{\prime} w^{\prime}}$ multiplied by the constant atmospheric background density $\bar{\varrho}_{0}$ at the reference level. Density however cancels out if accelerations $F$ (like $F_{\mathrm{KW}}$ above) are calculated (see Appendix A) and therefore the term momentum flux often is synonymously used for both $\overline{u^{\prime} w^{\prime}}$ and $\bar{\varrho}_{0} \overline{u^{\prime} w^{\prime}}$.

In our study we determine Kelvin wave momentum flux from the zonal wavenumber/frequency temperature spectra of SABER measurements and ECMWF analyses. For this purpose the spectral amplitudes of the temperature spectra are converted into momentum flux spectral amplitudes. This conversion is carried out by use of Eq. (10):

$\mathcal{F}_{p x}=\frac{\bar{\varrho}_{0}}{2} \frac{k^{2}}{N \hat{\omega}}|\widetilde{T}|^{2} /\left[\left(\frac{\gamma}{c_{s}^{2}}-\frac{1}{2 g H}\right)^{2}+\left(\frac{N k}{g \hat{\omega}}\right)^{2}\right]$

with $|\widetilde{T}|=\widehat{T} / T$, the temperature amplitude $\widehat{T}$ divided by the atmospheric background temperature $T$. Further, $k$ is the zonal wavenumber, $N$ the buoyancy frequency, $\hat{\omega}$ the intrinsic wave frequency, $\gamma$ the adiabatic coefficient, $c_{s}$ the sound speed, $g$ the gravity acceleration, and $H$ the pressure scale height. For a derivation of Eq. (10) see Appendix A.

\section{Average Kelvin wave temperature and momentum flux spectra}

\subsection{Kelvin wave temperature spectra}

To investigate the general behavior of temperature and momentum flux space-time spectra we average the spectra of all 31-day time windows over the period 1/2002 until 11/2006, 

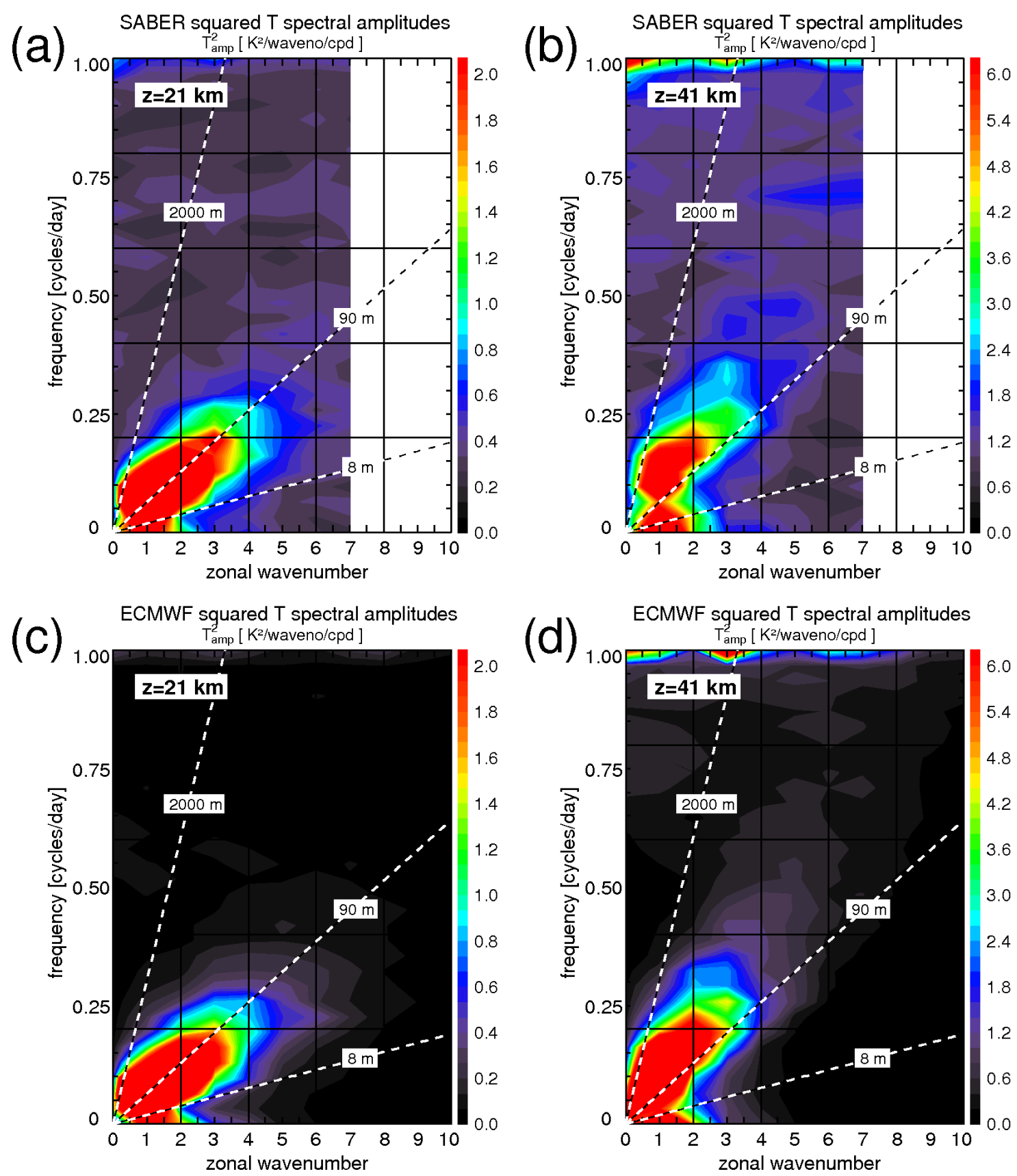

Fig. 1. Spectra of squared temperature amplitudes at the equator averaged over the whole time period 1/2002 until 11/2006 for SABER at $21 \mathrm{~km}$ (a) and $41 \mathrm{~km}$ (b) altitude. (c) and (d) show the same but for ECMWF. Values given are in units of $\mathrm{K}^{2} /$ wavenumber/(cycles/day). Also given are the lines for equivalent depths $h_{e}=8,90$, and $2000 \mathrm{~m}$, corresponding to ground based phase speeds of 9,30 , and $140 \mathrm{~m} / \mathrm{s}$.

similar as in Ern et al. (2008). Figure 1 shows these average temperature spectra at the equator for SABER at $21 \mathrm{~km}$ (Fig. 1a) and $41 \mathrm{~km}$ altitude (Fig. 1b). Figure 1c and d shows the same for ECMWF data. Different from Ern et al. (2008) the spectra are not split up into symmetric and antisymmetric spectra with respect to the equator and the spectra are not latitudinally averaged.
Although frequencies from -1 to 1 cycle/day can be resolved unambiguously by the satellite sampling we show only the range from 0 to 1 cycle/day because our work is focused on Kelvin waves, which propagate eastward and their spectral contribution is found at positive frequencies, and usually in the frequency range $0-1$ cycle/day. The zonal wavenumber range is selected from zero to 10 because spectral contributions of global scale equatorial waves are usually 
at those low wavenumbers. Please note that also only part of the full spectral range covered by the ECMWF data is shown. On the other hand the zonal wavenumber range of the SABER data is limited to wavenumbers lower than 6-7 due to the satellite sampling (see also Sect. 2.2). Therefore wavenumbers higher than 7 are left blank for SABER.

For comparison also the lines for equivalent depths of 8 , 90, and $2000 \mathrm{~m}$ are given for Kelvin waves (black and white dashed lines) under the assumption of zero background wind. These equivalent depths correspond to ground based phase speeds of about 9,30 , and $140 \mathrm{~m} / \mathrm{s}$, or vertical wavelengths of about 3, 9, and $50 \mathrm{~km}$ (also under the assumption of zero background wind).

Similar as in Ern et al. (2008) we find the pronounced lobeshaped spectral peak due to Kelvin waves in both SABER and ECMWF data. Although the spectra are not split into symmetric and antisymmetric contributions the Kelvin wave spectral peak is not significantly disturbed by contributions of any other equatorial wave mode. This is the case because Kelvin waves are the most dominant global scale wave mode in temperatures.

At both altitudes the most significant spectral contributions are found at zonal wavenumbers lower than about 4-5. As expected, the spectral peak is shifted towards higher ground based phase speeds and thus even lower zonal wavenumbers with altitude. This shift occurs because waves at low ground based phase speeds dissipate more and more with altitude, while at the same time the amplitudes of higher phase speed waves can grow with altitude without dissipation taking effect (see also Garcia and Salby, 1987; Salby and Garcia, 1987; Ern et al., 2008). Wave dissipation as mentioned above is even more effective because the QBO wind profile in the equatorial stratosphere exposes at any time zonal winds and vertical wind shears of zonal winds that will filter Kelvin waves of low phase speeds.

Like in Ern et al. (2008) we find qualitatively good agreement between SABER and ECMWF spectra. The only striking difference is the strong white noise spectral background in the SABER spectra. This spectral background is due to mesoscale gravity waves which are not resolved by the satellite sampling. The spectral background is lower in the ECMWF spectra because the ECMWF spectra cover a larger spectral region. In addition, the ECMWF model captures not all gravity waves present in the real atmosphere (only very long horizontal wavelength gravity waves). See also Ern et al. (2008) and Schroeder et al. (2009). In the following this white-noise spectral background will be subtracted to extract the true Kelvin wave signal in the spectra. The background is estimated like in Ern et al. (2008) by calculating the median of the spectral contributions at zonal wavenumbers 3 and higher (spectral peaks due to tides and stationary waves are also omitted).

For a cross check, we also carried out all analyses without subtracting the spectral background due to gravity waves, and we found that the results are almost unchanged for
ECMWF. For SABER, however, momentum fluxes are considerably higher if the background is not subtracted. Also accelerations can be considerably higher, especially for the high zonal wavenumbers $(k=4-6)$. The global relative structures of momentum fluxes and accelerations are however very similar to those with the spectral background subtracted.

\subsection{Kelvin wave momentum flux spectra}

As discussed in Sect. 2.3 we can obtain zonal wavenumber/frequency spectra of the vertical flux of horizontal momentum $\mathcal{F}_{p x}=\bar{\varrho}_{0} \overline{u^{\prime} w^{\prime}}$ for Kelvin waves by a transformation of the temperature space-time spectra via Eq. (10). Because this transformation is only valid for Kelvin waves the spectral background due to gravity waves is subtracted from the temperature spectra before the transformation (see above).

Figure 2 shows the resulting Kelvin wave momentum flux spectra averaged over the period 1/2002-11/2006 at the equator for SABER at $21 \mathrm{~km}$ (Fig. 2a) and $41 \mathrm{~km}$ altitude (Fig. 2b). Figure 2c and d shows the same for ECMWF data. Please note that the color scale is lower by a factor of 10 for the higher altitudes (Fig. 2b and d).

Again, we find qualitatively good agreement between SABER and ECMWF spectra. Compared with the temperature spectra higher zonal wavenumbers play a more important role in momentum flux spectra (see also Tindall et al., 2006a,b). This is the case because in temperature spectra the Kelvin wave spectral feature is lobe-shaped, approximately along the lines of constant phase velocities (i.e., along lines of constant equivalent depth). For constant intrinsic phase velocity $\hat{c}_{\phi}=\hat{\omega} / k$ the scaling factor between squared temperature amplitudes and momentum flux, given in Eq. (10), is proportional to $k$, the zonal wavenumber. Therefore high zonal wavenumbers are more enhanced in momentum flux spectra than in temperature spectra.

However some spectral features at high zonal wavenumbers and high frequencies, for example the spectral feature at zonal wavenumbers 6-7 and a frequency of about 0.7 cycles/day in Fig. $2 b$, are likely not spectral contributions due to Kelvin waves. These features are well separated from the lobe-shaped Kelvin wave spectral peak and could be due to $\mathrm{n}=1$ inertia-gravity waves, due to some minor spectral aliasing, or due to some remains of the gravity wave spectral background which are strongly enhanced by the transformation into momentum flux. Nevertheless, the bulk of momentum flux is still found at zonal wavenumbers $\leq 6$ and frequencies $<0.4$ cycles/day for both SABER and ECMWF.

The ECMWF operational analyses rely strongly on the assimilation of measured satellite data. SABER data are not assimilated into ECMWF, but, for example, TOVS/ATOVS nadir radiances are assimilated (e.g., Li et al., 2000). These data sets also contain information of zonal wavenumbers higher than six because the TOVS/ATOVS radiances provide information across the satellite track due to the large swath width of about $1500 \mathrm{~km}$ across track. Therefore also 

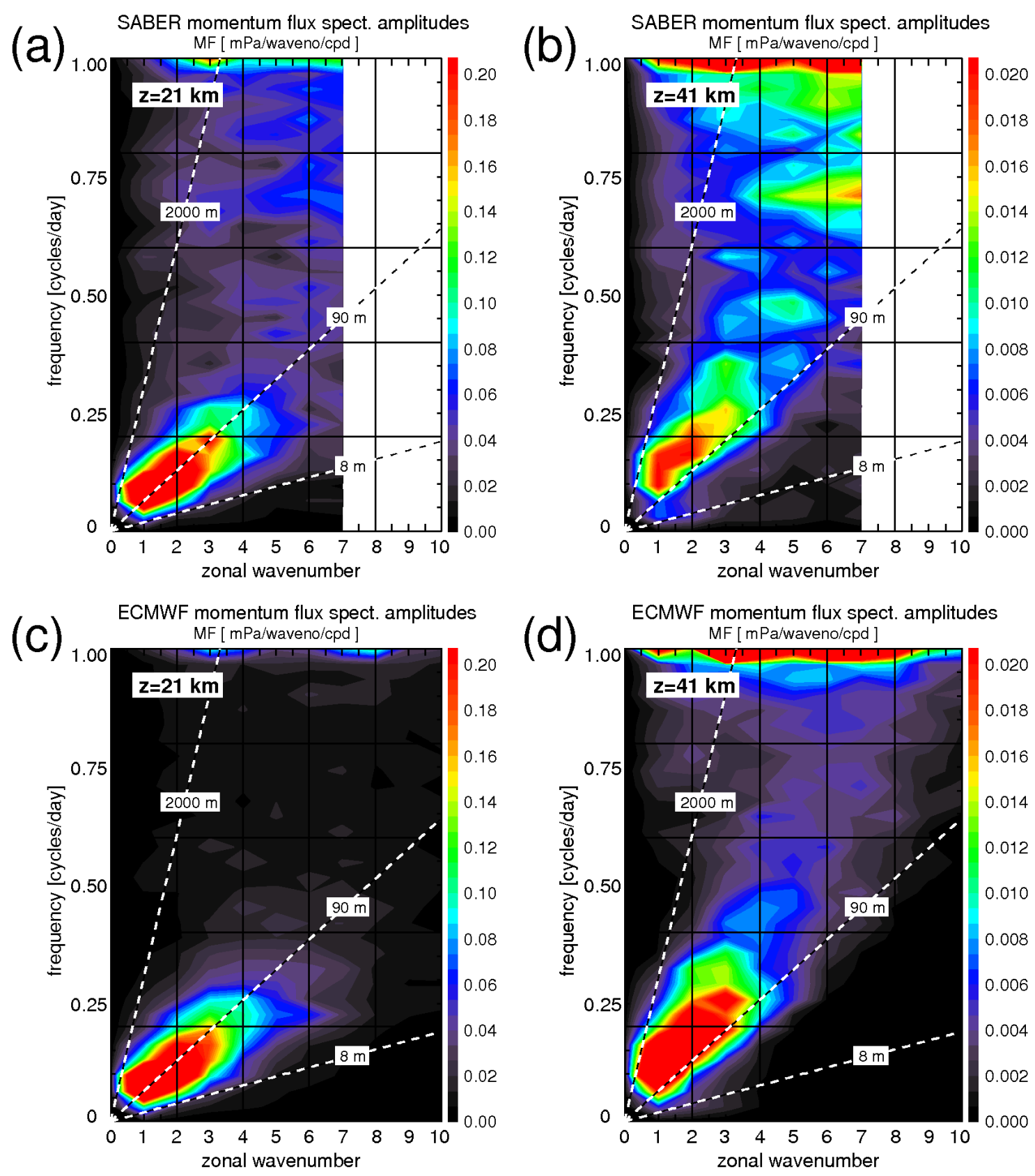

Fig. 2. Same as Fig. 1 but for the vertical flux of zonal momentum due to Kelvin waves. Units are $10^{-3} \mathrm{~Pa} /$ wavenumber/(cycles/day).

the ECMWF analyses should contain some contributions of equatorial wave modes at zonal wavenumbers higher than 6 , if present.

In the average ECMWF temperature, as well as momentum flux spectra above about $21 \mathrm{~km}$ altitude (see Figs. 1c and 2c) it looks like these high zonal wavenumber contributions on average already have dissipated at lower altitudes and the most relevant spectral contributions are at zonal wavenumbers $\leq 6$. This means that the bulk of Kelvin wave momentum flux can, indeed, be estimated from low Earth orbit satellite measurements (like the SABER measurements) providing information only for zonal wavenumbers lower than about 7 due to the satellite orbit geometry.

\subsection{Altitude-time cross sections of Kelvin wave temper- ature variances and momentum flux}

From Figs. 1 and 2 we can also see that the Kelvin wave spectral contribution is a localized spectral feature in both temperature and momentum flux spectra. This means that 

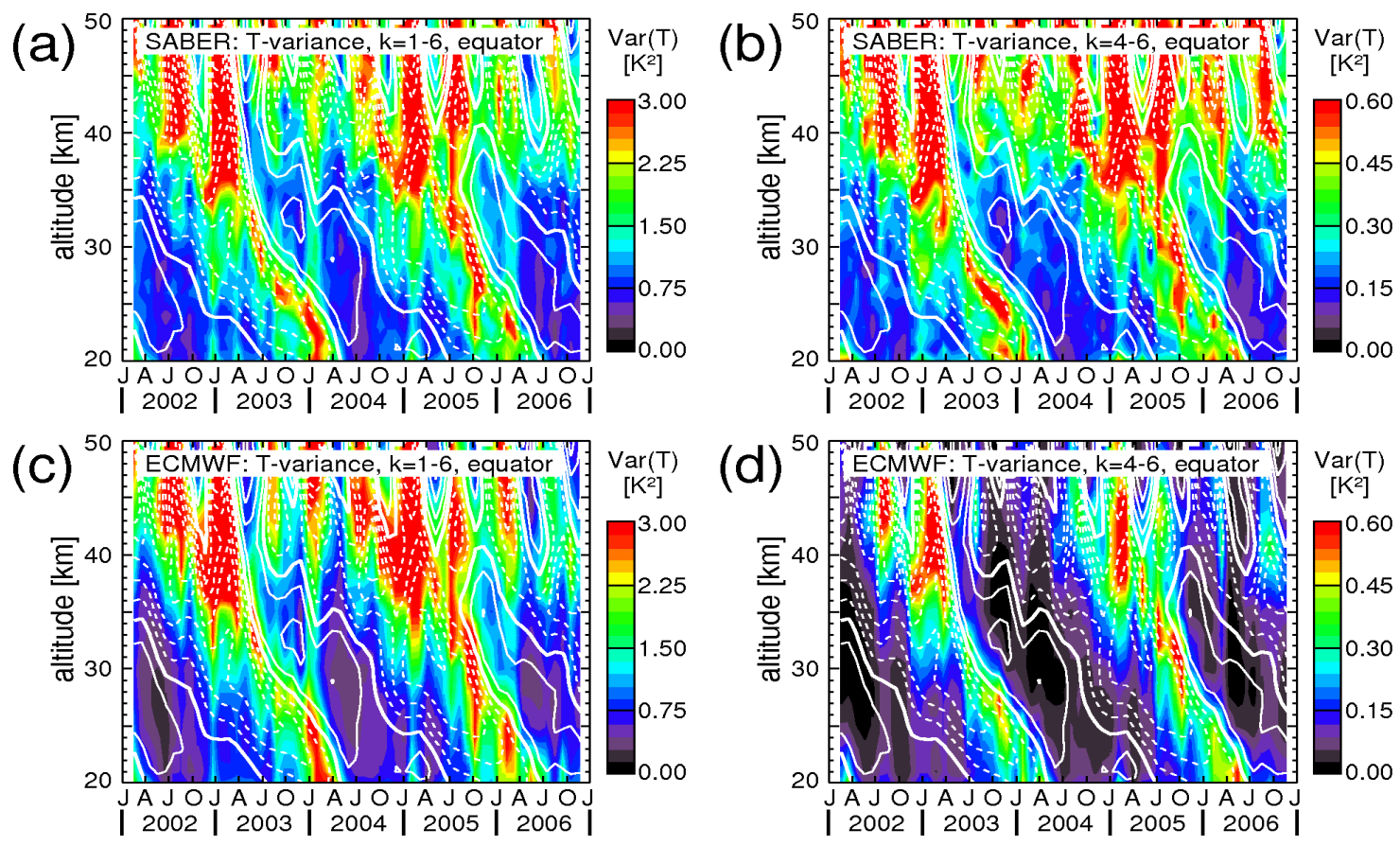

Fig. 3. Altitude-time cross sections of Kelvin wave temperature variances in $\mathrm{K}^{2}$ at the equator for SABER in the full wave band $k=1-6$ (a) and for high zonal wavenumbers 4-6 only (b). (c) and (d) show the same but for ECMWF. For comparison also the zonal mean zonal wind at the equator taken from ECMWF averaged over the 31-day time windows is shown as white contour lines. Contour increment is $10 \mathrm{~m} / \mathrm{s}$. The bold solid line is the zero wind line, easterlies are indicated by dashed and westerlies by solid contour lines.

wave bands similar as in Ern et al. (2008) can be defined to obtain temperature variances as well as momentum flux values for Kelvin waves by integrating over those spectral bands.

We define the following two wave bands: The first wave band comprises the whole contribution due to Kelvin waves. This wave band covers zonal wavenumbers 1-6 and the whole range between the lines of 8 and $2000 \mathrm{~m}$ equivalent depth shown in Figs. 1 and 2. In addition, a high-frequency cutoff at 0.4 cycles/day is introduced like in Ern et al. (2008). Values obtained by integrating over this wave band will, for example, be called "total" temperature variance or "total" momentum flux of Kelvin waves. To investigate the relevance of high zonal wavenumbers we define another wave band which covers the same frequency range as the "total" Kelvin wave band, but only zonal wavenumbers 4-6.

The high frequency cutoff at 0.4 cycles/day is chosen to make sure that all spectral contributions are due to Kelvin waves. Below about 0.4 cycles/day the contribution of Kelvin waves is very strong and likely not distorted by other equatorial wave modes. At higher frequencies, however, also other global scale equatorial wave modes, especially inertiagravity waves (see also Ern et al., 2008), might contribute to the spectral amplitudes found.

Nevertheless, from Figs. 1 and 2 one has the impression that the lobe-shaped spectral peak due to Kelvin waves extends further towards frequencies as high as about 0.60.8 cycles/day. For a cross-check we therefore also carried out all analyses with a high frequency cutoff at 0.6 cycles/day and 0.8 cycles/day without showing the results. For a cutoff at 0.6 cycles/day momentum fluxes and accelerations derived are somewhat higher (about 10-20\% for zonal wavenumbers 1-6) than the results presented in the following sections. Higher zonal wavenumbers $(k=4-6)$ are even somewhat more enhanced. The global relative structures are however unchanged. With a high frequency cutoff at 0.8 cycles/day derived momentum fluxes and accelerations are even higher, but the global distributions show an enhanced level of noise.

In the following all results presented will be based on a high frequency cutoff at 0.4 cycles/day. Figure 3 shows altitude-time cross sections of Kelvin wave temperature variances at the equator in the total (zonal wavenumbers 1-6) and the high zonal wavenumber $(k=4-6)$ wave bands discussed above for SABER (Fig. 3a and b) and ECMWF (Fig. 3c and d).

Obviously, the temperature variances contained in the high zonal wavenumber spectral band are considerably lower (about a factor of 5) than in the full spectral band for both SABER (Fig. 3a and b) and ECMWF (Fig. 3c and d). This means that about $80 \%$ of the variances are located at the low zonal wavenumbers $1-3$. Please note that the color scale of Fig. $3 b$ and $d$ is different from the one in Fig. $3 a$ and c. The 

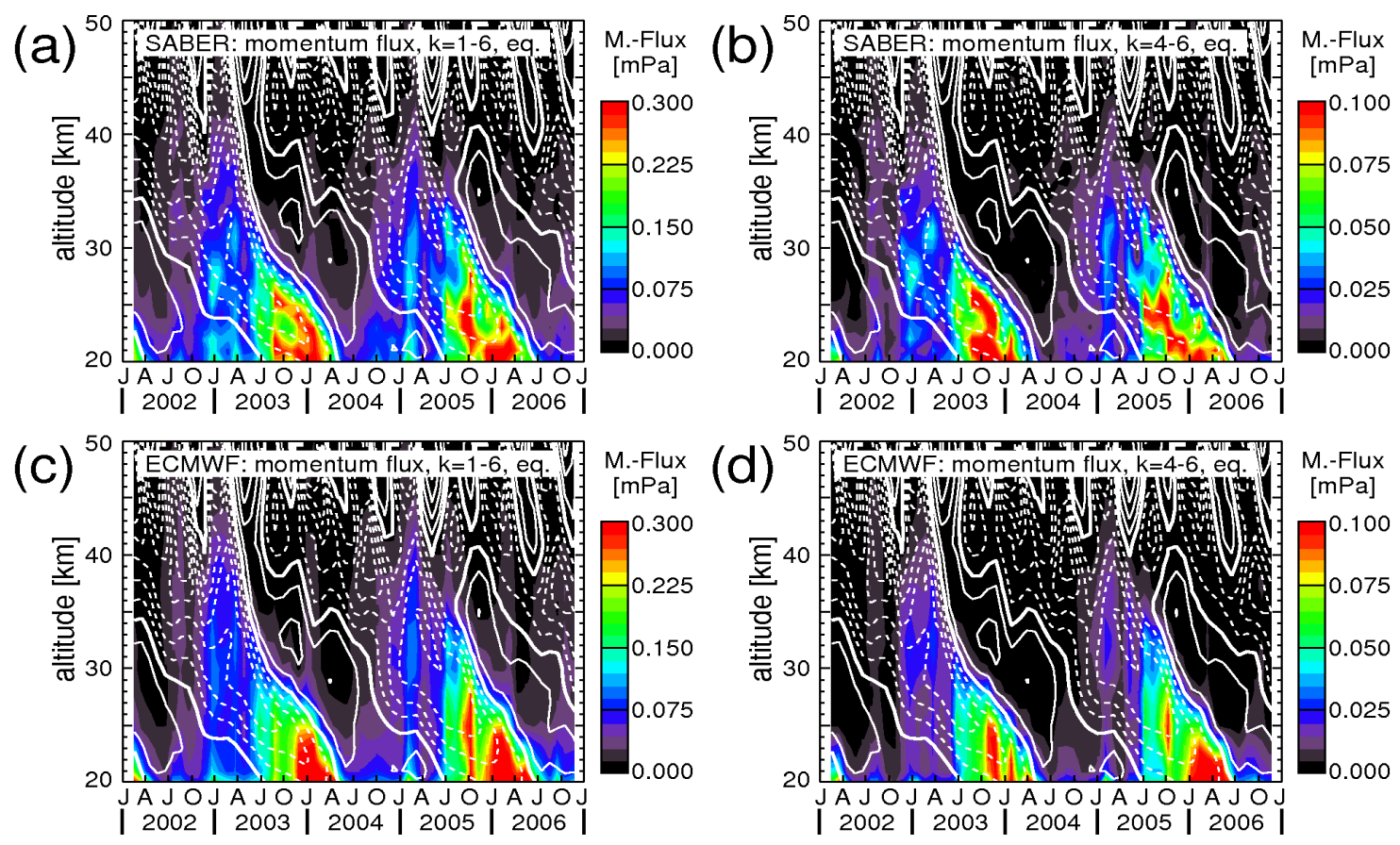

Fig. 4. Altitude-time cross sections of Kelvin wave momentum flux in units of $10^{-3} \mathrm{~Pa}(\mathrm{mPa})$ at the equator for SABER in the full wave band $k=1-6$ (a) and for high zonal wavenumbers 4-6 only (b). (c) and (d) show the same but for ECMWF. For comparison also the ECMWF zonal mean zonal wind at the equator averaged over the 31-day time windows is shown as white contour lines. Contour increment is $10 \mathrm{~m} / \mathrm{s}$. The bold solid line is the zero wind line, easterlies are indicated by dashed and westerlies by solid contour lines.

relative structure of the variances at high zonal wavenumbers is however the same as for the total variances. There is good agreement between SABER and ECMWF for both the total as well as the high wavenumber variances.

Figure 4 shows altitude-time cross sections of Kelvin wave momentum flux in units of $10^{-3} \mathrm{~Pa}$ (i.e., milli-Pascal (mPa)) at the equator in the two wave bands discussed above for SABER (Fig. 4a and b) and ECMWF (Fig. $4 \mathrm{c}$ and d). For comparison again the zonal mean wind at the equator taken from ECMWF averaged over the 31-day time windows is shown as white contour lines.

We expect that the momentum flux $\mathcal{F}_{p x}=\bar{\varrho}_{0} \overline{u^{\prime} w^{\prime}}$ should be constant if the waves propagate conservatively and no wave sources are present. Only if the waves dissipate (i.e., interact with the background atmosphere) the momentum flux decreases. Momentum flux should be highest at the source altitudes. Since Kelvin waves are generated in the troposphere we expect momentum flux to decrease monotonically with altitude in the stratosphere. This is also what we find in all panels of Fig. 4, i.e. for all wave bands and both SABER and ECMWF. Momentum flux is about constant with altitude. Only if the waves encounter positive vertical wind shear momentum flux decreases.

Different from Fig. 3 the contribution at high zonal wavenumbers $(k=4-6)$ is now no longer negligible. It is about $30 \%$ of the total momentum flux. We again find good agreement between SABER and ECMWF. The contribution of zonal wavenumbers $k=3-6$ (not shown) is even higher (about $50 \%$ of the total momentum flux due to Kelvin waves).

Figure 5 shows the same as Fig. 4 but averaged over the whole latitude band from $10^{\circ} \mathrm{S}-10^{\circ} \mathrm{N}$. In about this latitude band Kelvin wave forcing of the background winds takes effect. This is why values of Kelvin wave momentum flux and acceleration averaged over this latitude band are a relevant quantity to judge the overall effect of Kelvin waves in global dynamics. Therefore also other authors use this latitudinal average in their work (e.g., Hitchman and Leovy, 1988; Kawatani et al., 2009) and we additionally provide the values given in Fig. 5 as a reference for the comparison with GCM results as well as previous estimates (see next subsection).

We find that the latitudinally averaged momentum flux is somewhat lower than the equatorial value. The relative structures are however the same. And again, there is good agreement between SABER and ECMWF.

\subsection{Comparison of SABER and ECMWF Kelvin wave momentum fluxes with previous estimates}

As has been discussed by Ern et al. (2008) the Kelvin wave temperature variances of SABER and ECMWF are in good agreement with previous observations. We will now also 

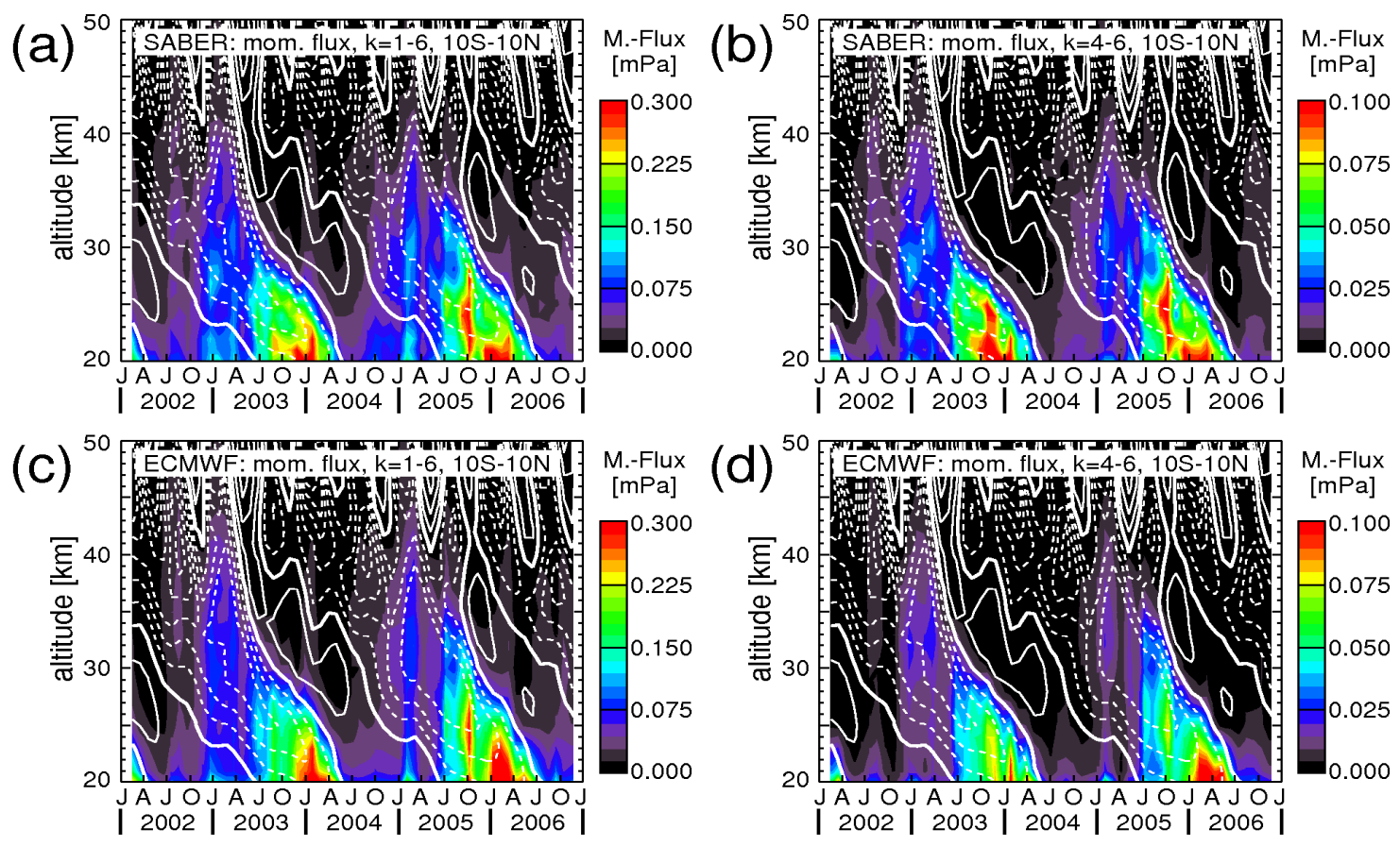

Fig. 5. Same as Fig. 4 but momentum flux as well as zonal wind contours are averages over the latitude band $10^{\circ} \mathrm{S}-10^{\circ} \mathrm{N}$.

compare the momentum flux values derived from SABER and ECMWF space-time spectra with other observations.

Our values of total Kelvin wave momentum flux are about 0.3-0.4 mPa during periods of strong Kelvin wave activity in the altitude range $20-24 \mathrm{~km}$ at the equator. Peak values are somewhat lower, about $0.3 \mathrm{mPa}$, if averaged over the latitude band $10^{\circ} \mathrm{S}-10^{\circ} \mathrm{N}$. Values decrease gradually with altitude. For example, peak values at $30 \mathrm{~km}$ altitude have decreased to about $0.08-0.12 \mathrm{hPa}$ for the total Kelvin wave momentum fluxes averaged over $10^{\circ} \mathrm{S}-10^{\circ} \mathrm{N}$ (see Fig. 5a and c).

There are several estimates directly based on observational data (satellites as well as rawinsondes). For example, Hitchman and Leovy (1988) estimated from Limb Infrared Monitor of the Stratosphere (LIMS) satellite data an average value of about $0.07 \mathrm{mPa}$ for zonal wavenumbers $1-3$ averaged over several months of strong Kelvin wave activity and latitudes $10^{\circ} \mathrm{S}-10^{\circ} \mathrm{N}$ at $10 \mathrm{hPa}$ (about $32.3 \mathrm{~km}$ altitude). This is in very good agreement with our peak values of $0.08-0.12 \mathrm{mPa}$ for total momentum flux at $30 \mathrm{~km}$ altitude, if we take into account that about one third of the momentum flux is located at zonal wavenumbers higher than three and is not contained in the estimates by Hitchman and Leovy (1988).

Dunkerton (1997) estimated for long period waves with an average period of about 15 days momentum flux of $6 \times 10^{-3} \mathrm{~m}^{2} / \mathrm{s}^{2}$ at $7^{\circ} \mathrm{N}$ and about $20-25 \mathrm{~km}$ altitude during periods of enhanced Kelvin wave activity. This estimate is based on the observations by Wallace and Kousky (1968). If multiplied by atmospheric density this value corresponds to momentum flux values of about $0.35 \mathrm{mPa}$. This is in good agreement with our peak values in the lower stratosphere.
Another estimate derived from rawinsondes at Singapore (i.e., close to the equator at $1^{\circ} \mathrm{N}$ ) by Sato and Dunkerton (1997) is about $4-12 \times 10^{-3} \mathrm{~m}^{2} / \mathrm{s}^{2}$ (about $0.15-0.6 \mathrm{mPa}$ ) at about $25 \mathrm{~km}$ altitude for long period waves with periods longer than 3 days during westerly wind shear (i.e., most likely Kelvin waves). This estimate is also in good agreement with our peak values in the lower stratosphere.

The flux due to short period waves (periods shorter than 3 days, likely mesoscale gravity waves) determined by Sato and Dunkerton (1997) is considerably higher. Estimates are about $20 \times 10^{-3} \mathrm{~m}^{2} / \mathrm{s}^{2}$ at $21 \mathrm{~km}$ and $50 \times 10^{-3} \mathrm{~m}^{2} / \mathrm{s}^{2}$ at $30 \mathrm{~km}$ altitude (about $1.5 \mathrm{mPa}$ at $21 \mathrm{~km}$ and $1 \mathrm{mPa}$ at $30 \mathrm{~km}$ altitude) during periods of westerly wind shear. During periods of easterly wind shear values are similar (about 7$30 \times 10^{-3} \mathrm{~m}^{2} / \mathrm{s}^{2}$ ). Momentum fluxes due to mesoscale gravity waves that are considerably higher than the fluxes of Kelvin waves were also found by Ern et al. (2004, 2006). Absolute values of gravity wave momentum flux of about $1-2 \mathrm{mPa}$ were derived in the tropics at about $25 \mathrm{~km}$ altitude from Cryogenic Infrared Spectrometers and Telescopes for the Atmosphere (CRISTA) satellite data.

Momentum fluxes $\overline{u^{\prime} w^{\prime}}$ likely due to Rossby-gravity waves were determined by Maruyama (1994) during phases of westerly QBO winds. Values are about $2-8 \times 10^{-3} \mathrm{~m}^{2} / \mathrm{s}^{2}$ at all times near $17 \mathrm{~km}$ altitude (about $0.3-1.1 \mathrm{mPa}$ ). The lower limit of the range is more realistic, and for the calculation of wind acceleration for Rossby-gravity waves also meridional heat fluxes have to be taken into account (Dunkerton, 1997). 
Momentum fluxes were also obtained from the ECMWF ERA-15 reanalysis data by Tindall et al. (2006a). For Kelvin waves average values are $1.3,1.7$, and $0.9 \mathrm{~m}^{2} / \mathrm{s}^{2}$ at the ERA15 pressure levels 100,70 , and $50 \mathrm{hPa}$, respectively. These values correspond to about $0.2,0.2$, and $0.07 \mathrm{mPa}$ at altitudes of about 16.2, 18.7, and $21.1 \mathrm{~km}$, respectively. Momentum flux values for Rossby-gravity and equatorial Rossby waves are about an order of magnitude lower. All the values by Tindall et al. (2006a) are averaged over 15-years and over the latitude band $7.5^{\circ} \mathrm{S}-7.5^{\circ} \mathrm{N}$. If we compare these average values with our $10^{\circ} \mathrm{S}-10^{\circ} \mathrm{N}$ averages, we find that the values at about $21 \mathrm{~km}$ altitude by Tindall et al. (2006a) are about $20 \%$ of our maximum values during periods of strong Kelvin wave activity. This is also good agreement taking into account the temporal averaging by Tindall et al. (2006a), but might be somewhat lower than our values.

This means that our results for Kelvin wave momentum fluxes are in reasonable agreement with the previous findings by Hitchman and Leovy (1988), Dunkerton (1997), Sato and Dunkerton (1997), and Tindall et al. (2006a).

It should be noted that SABER temperature variances and also momentum flux values show some minor oscillations with altitude, which is not as expected from wave theory. This effect will be discussed in more detail in Sect. 4 when zonal wind accelerations due to Kelvin waves are determined.

\section{Zonal wind acceleration}

\subsection{Wind acceleration terms derived from an ECMWF background atmosphere}

In Sect. 4.2 we want to quantify the relative contribution of Kelvin waves to the total wave forcing balancing the zonal mean zonal flow of the background atmosphere. Because part of the wave forcing is not properly resolved by measurements and models an estimate of the total wave forcing can only be provided by quantifying the different acceleration terms of the background atmosphere. According to Eq. (6) in Sect. 2.3 there are three zonal wind acceleration terms of the background atmosphere that are relevant. First, there is the tendency of the zonal wind $\partial \bar{u} / \partial t$. Second, there is the meridional advection term $\bar{v}(\partial \bar{u} / \partial y-f)$, and, third, there is the vertical advection term $\bar{w} \partial \bar{u} / \partial z$.

These terms are estimated based on an ECMWF background atmosphere averaged zonally as well as temporally over the 31-day time windows of our analyses. The results are shown in Fig. 6 at the equator for the zonal wind tendency (a), the horizontal advection term (b), and the vertical advection term (c). Figure $6 \mathrm{~d}$ shows the sum of all three terms, representing an estimate of the total wave forcing. The values given are in units of $\mathrm{m} / \mathrm{s} /$ day. Figure $6 \mathrm{e}-\mathrm{h}$ shows the same as Fig. $6 \mathrm{a}-\mathrm{d}$ but averaged over the latitude band $10^{\circ} \mathrm{S}-10^{\circ} \mathrm{N}$.
One of the largest terms in Fig. 6 is the zonal wind tendency (see Fig. 6a and e) because of the strong temporal gradients of the QBO and SAO winds. But also the meridional advection terms can be quite strong (see Fig. $6 \mathrm{~b}$ and $\mathrm{f}$ ). This is mainly caused by meridional gradients of the zonal mean zonal wind at altitudes above about $35 \mathrm{~km}$. But in the $10^{\circ} \mathrm{S}-10^{\circ} \mathrm{N}$ averages in Fig. $6 f$ there are also considerable accelerations due to the meridional advection term even below $35 \mathrm{~km}$ altitude. Also the vertical advection term can sometimes attain values as strong as -0.5 or $+0.25 \mathrm{~m} / \mathrm{s} /$ day. Therefore the total acceleration of the zonal mean zonal wind (see Fig. 6d and h) is the delicate result of the sum of three terms that can all be of the same order.

One of the larger error sources is the zonal mean vertical velocity with values as small as about $1 \mathrm{~mm} / \mathrm{s}$. Experimental values of the residual mean vertical velocity have been determined, for example, by Dunkerton (1997). His values are about $0.5 \mathrm{~mm} / \mathrm{s}$ at about $16 \mathrm{~km}$ altitude, attain a minimum value of $0.2-0.3 \mathrm{~mm} / \mathrm{s}$ at about $20 \mathrm{~km}$ altitude, and are $0.5-0.6 \mathrm{~mm} / \mathrm{s}$ at about $30 \mathrm{~km}$ altitude. Similar values were also derived by Schoeberl et al. (2008). For comparison, zonal mean ECMWF values averaged over the whole period $1 / 2002-11 / 2006$ and $10^{\circ} \mathrm{S}-10^{\circ} \mathrm{N}$ are about $0.8 \mathrm{~mm} / \mathrm{s}$ at $16 \mathrm{~km}$ altitude, attain a minimum value of about $0.3 \mathrm{~mm} / \mathrm{s}$ at about $21 \mathrm{~km}$ altitude, and are about $0.4 \mathrm{~mm} / \mathrm{s}$ at about $30 \mathrm{~km}$ altitude. This means that there are some discrepancies, but the ECMWF values are realistic enough to provide a rough estimate of the vertical advection term.

Nevertheless the total acceleration of the zonal mean zonal wind will be subject to large errors and will also be strongly dependent on the data set or model used. Values therefore have to be treated with caution. While the zonal wind tendency can be estimated with reasonable accuracy, the advection terms are more uncertain. This is the case because the zonal average meridional and vertical velocities are of the order of only about $1 \mathrm{~m} / \mathrm{s}$ and about $1 \mathrm{~mm} / \mathrm{s}$, respectively.

Therefore our estimates of the total background acceleration will be most reliable at the equator at altitudes below about $35 \mathrm{~km}$ where the advection terms are small. This is the case because on one hand the Coriolis parameter $f$ is zero at the equator and also meridional gradients in the zonal wind are small in the lower stratosphere at the equator. On the other hand vertical velocities are minimum in the altitude region around $20-25 \mathrm{~km}$.

Uncertainties of the background acceleration terms are however not relevant for the determination of the Kelvin wave accelerations in the next subsection. They are only important for quantifying the relative contribution of Kelvin waves to the total wave forcing. 

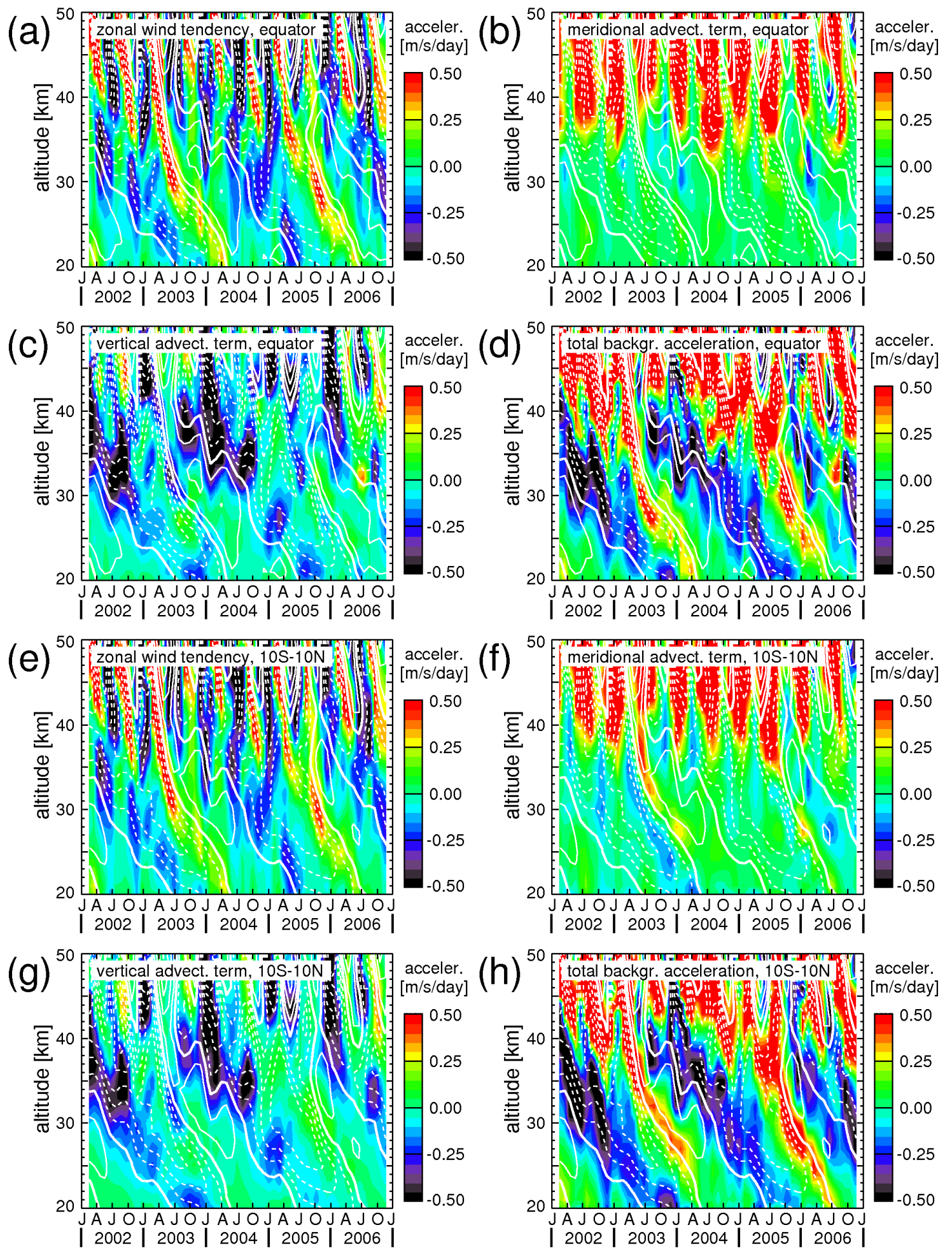

Fig. 6. Zonal mean zonal wind accelerations derived for an ECMWF background atmosphere which is averaged both zonally and over the 31-day time windows of our analysis. Results for the equator are given for the zonal wind tendency (a), the meridional advection term (b), and the vertical advection term (c). The total forcing of the background atmosphere (i.e., the sum of a-c) is given in (d). (e-h) shows the same but averaged over the latitude band $10^{\circ} \mathrm{S}-10^{\circ} \mathrm{N}$. Values given are in units of $\mathrm{m} / \mathrm{s} / \mathrm{day}$. Like in Figs. 4 and 5 for comparison the white contour lines indicate the zonal mean zonal wind. 

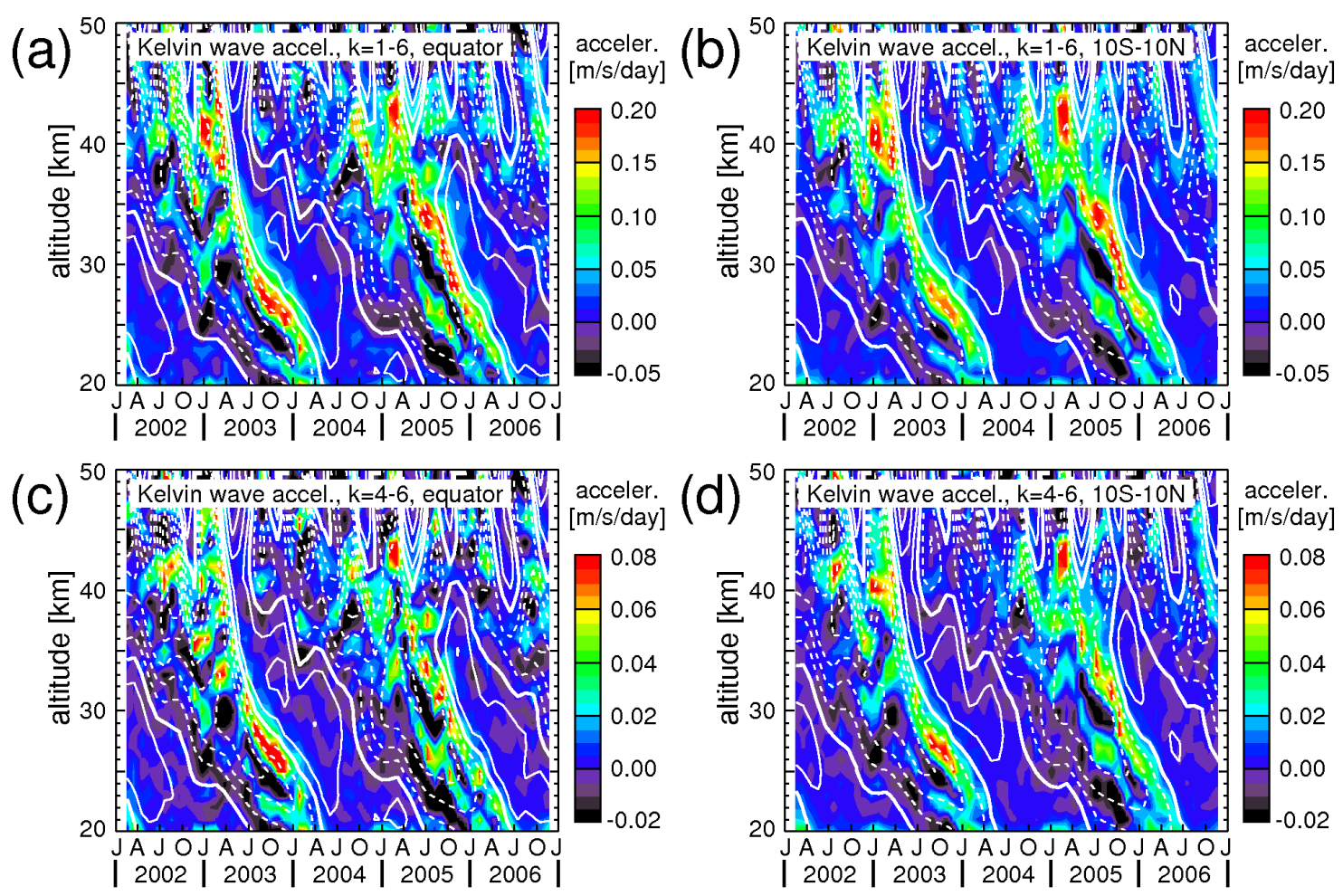

Fig. 7. Altitude time cross sections of zonal wind accelerations due to Kelvin waves derived from SABER space-time spectra. (a) is the total $(k=1-6)$ Kelvin wave acceleration at the equator and (b) averaged over the latitude band $10^{\circ} \mathrm{S}-10^{\circ} \mathrm{N}$. Accelerations due to high zonal wavenumber $(k=4-6)$ Kelvin waves are also given in (c and d). Values given are in units of $\mathrm{m} / \mathrm{s} /$ day. Again, for comparison the contour lines of the zonal mean zonal wind taken from ECMWF are shown.

\subsection{Kelvin wave zonal wind acceleration}

\subsubsection{Vertical smoothing of SABER temperature vari- ances}

Zonal wind accelerations due to Kelvin waves are derived by calculating the vertical gradients of the vertical flux of horizontal momentum $\mathcal{F}_{p x}$ (see Eq. 8 in Sect. 2.3). Accelerations are calculated from total $(k=1-6)$ Kelvin wave momentum flux values as well as for high zonal wavenumbers $k=4-6$ only (see Sect. 3.3). Altitude-time cross sections of total Kelvin wave accelerations $(k=1-6)$ are shown in Fig. 7 for SABER at the equator (Fig. 7a) and averaged over the latitude band $10^{\circ} \mathrm{S}-10^{\circ} \mathrm{N}$ (Fig. 7b). The contribution of high zonal wavenumber $(k=4-6)$ Kelvin waves is also shown in Fig. 7c and Fig. 7d.

As already indicated in the SABER Kelvin wave momentum fluxes shown in Figs. 4 and 5 there is also an oscillation in the vertical profiles of SABER accelerations in Fig. 7, and there are both positive and negative accelerations. For example, this is very prominent in the second half of the year 2005 when a pronounced double-peak structure is found for altitudes $25-35 \mathrm{~km}$ and significant negative values in between. This behavior is not expected from theory. Accelerations due to Kelvin waves should always be positive because they can only accelerate the background wind into direction of their phase speed, which is eastward for Kelvin waves.

One possible reason for these oscillations could be artifacts that can occur during the retrieval of atmospheric temperatures. The SABER temperatures are derived from measured radiance altitude profiles of the $15 \mu \mathrm{m}$ emission of $\mathrm{CO}_{2}$. Measurement technique and retrieval setup are, for example, described in Remsberg et al. (2004) and Remsberg et al. (2008). Radiances are measured with a step-width of $400 \mathrm{~m}$ vertically, while the instantaneous vertical field-ofview is about $2 \mathrm{~km}$. This means that there is an oversampling in the vertical direction. The retrieval procedure of the V1.06 temperatures used in our study is based on an onion peeling method. The additional information obtained by the vertical oversampling is accounted for in the retrieval algorithm. Five altitude profiles are derived independently using a retrieval step-width of about $2 \mathrm{~km}$ and only every fifth radiance value vertically. These five altitude profiles offset vertically by about $400 \mathrm{~m}$, each, are finally combined to the resulting altitude profiles.

The accurate measurement of dissipating waves, like Kelvin waves which encounter strong vertical wind shear, is a challenge for every retrieval of temperatures from radiance profiles measured from satellites. While there is a strong wave signal at one altitude the signal can completely 

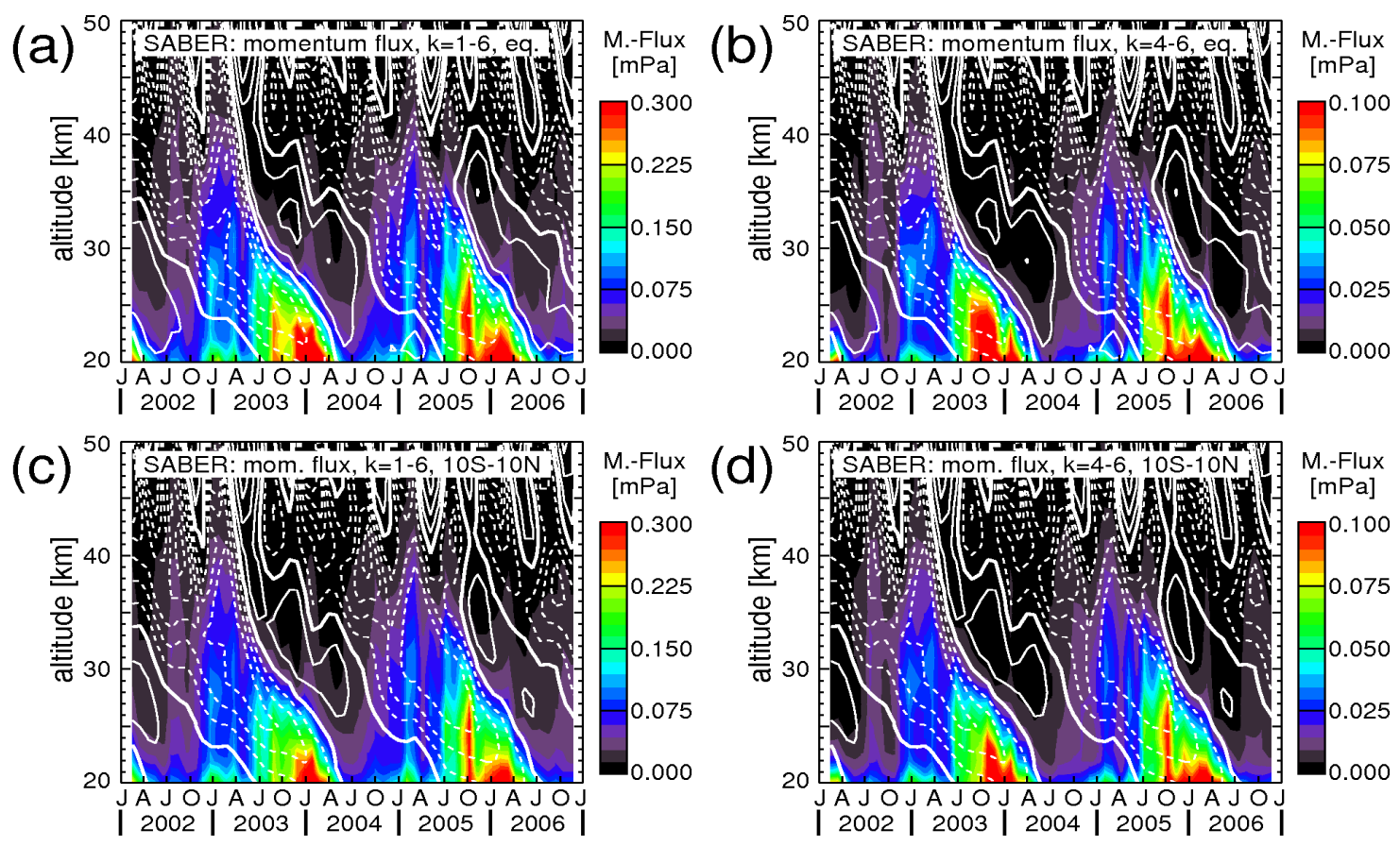

Fig. 8. Altitude-time cross sections of Kelvin wave momentum flux in units of $10^{-3} \mathrm{~Pa}(\mathrm{mPa})$ derived from SABER temperature variances smoothed vertically by a 4-km running mean to remove the oscillations present in Figs. 4 and 5. Values shown are for the equator in the full wave band $k=1-6$ (a) and for high zonal wavenumbers 4-6 only (b). (c) and (d) show the same but averaged over the latitude band $10^{\circ} \mathrm{S}-$ $10^{\circ} \mathrm{N}$. For comparison in (a) and (b) also the ECMWF zonal mean zonal wind at the equator and in (c) and (d) averaged over $10^{\circ} \mathrm{S}-10^{\circ} \mathrm{N}$ is shown as white contour lines.

disappear only $2 \mathrm{~km}$ above due to wave dissipation. This can, for example, frequently be found in Fig. 3.

It is possible that such strong gradients in wave activity in an altitude profile could induce small artificial oscillations in the temperature values derived. While not very relevant for the temperature variances there can be strong effects when vertical gradients of the variances are calculated like in our analysis. These oscillations will most likely have a dominant period at about two times the step-width of the retrieval algorithm. Therefore we smooth the SABER temperature variances vertically with a $4-\mathrm{km}$ running mean to reduce the oscillations found and to obtain more realistic momentum fluxes and accelerations.

Momentum fluxes derived from vertically smoothed SABER temperature variances are shown in Fig. 8. Obviously the vertical oscillations are removed almost completely. Therefore it is also expected that oscillations in the Kelvin wave accelerations are strongly reduced and the further discussion of SABER Kelvin wave accelerations will be based on the vertically smoothed data (see the next subsection).

\subsubsection{Kelvin wave accelerations derived from SABER and ECMWF temperature variances}

Kelvin wave accelerations resulting from vertically smoothed SABER temperature squared amplitudes for the same cases as in Fig. 7a-d are shown in Fig. 9a-d. We find that, indeed, the oscillations are strongly reduced by the vertical smoothing. Negative accelerations are removed almost completely.

In addition, in Fig. 9e and $\mathrm{f}$ also the relative contribution of the total Kelvin wave acceleration in percent with respect to the total wave forcing represented by the sum of the acceleration terms of the ECMWF background atmosphere (see Fig. 6) is given. For reference also the total acceleration of the background atmosphere (i.e., the estimate for the total wave forcing) is shown in Fig. $9 \mathrm{~g}$ for the equator and in Fig. $9 \mathrm{~h}$ averaged over the latitude band $10^{\circ} \mathrm{S}-10^{\circ} \mathrm{N}$. Additionally, in each panel for comparison the contour lines of the zonal mean zonal wind taken from ECMWF are shown. Please note that where applicable the ECMWF background atmosphere is meridionally smoothed to provide representative values for the broader latitudinal bins of the SABER data. Figure 10 shows the same as Fig. 9, but for the ECMWF Kelvin waves. 

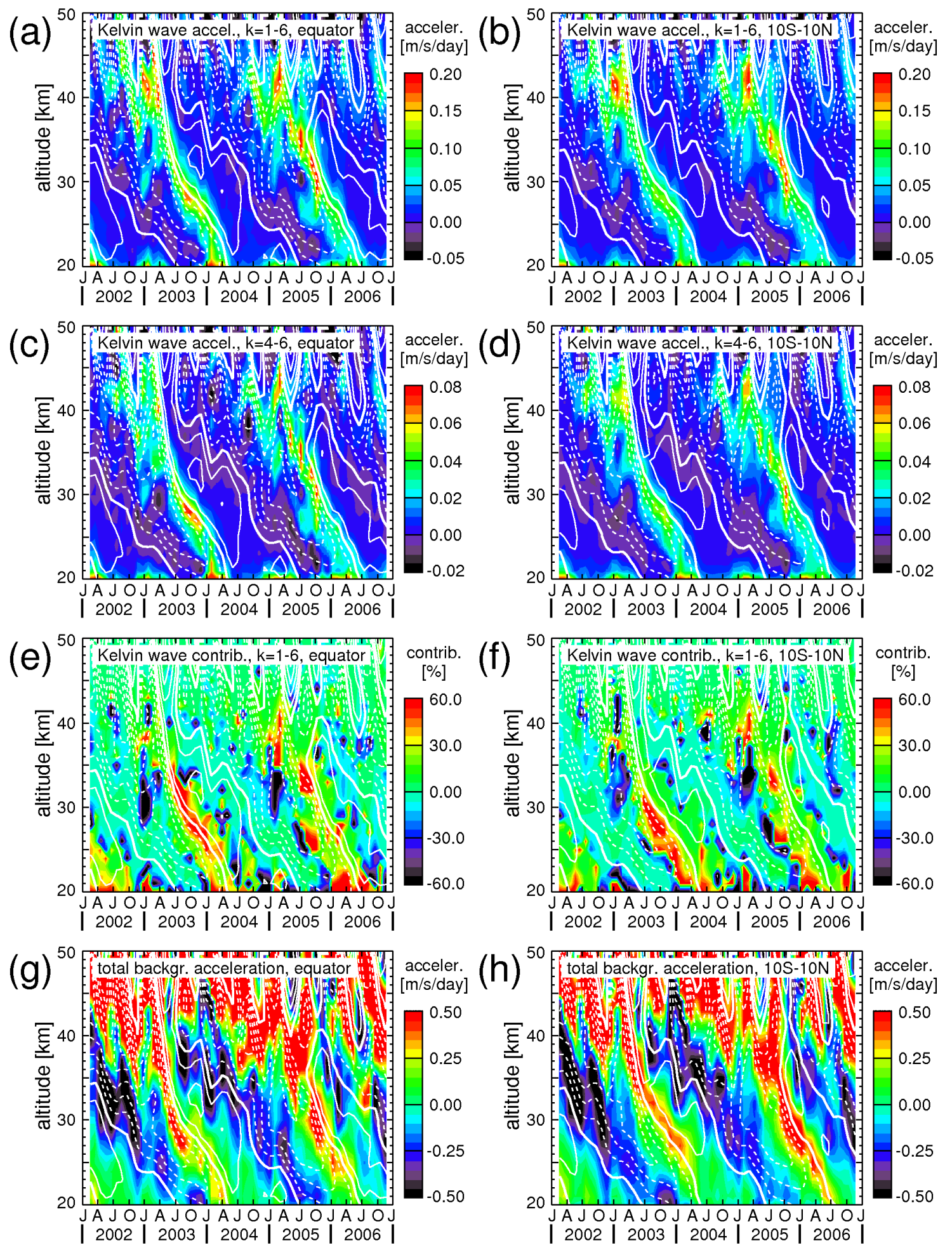

Fig. 9. Accelerations shown in Panels (a)-(d) are the same as in Fig. 7, but the SABER squared temperature amplitudes were smoothed vertically by a 4-km running mean to remove the oscillations present in Fig. 7. Additionally shown are the relative contribution of the total Kelvin wave acceleration in percent (Fig. 9e and 9f) with respect to the sum of the acceleration terms of the ECMWF background atmosphere given for comparison in Fig. $9 \mathrm{~g}$ at the equator and in Fig. $9 \mathrm{~h}$ averaged over $10^{\circ} \mathrm{S}-10^{\circ} \mathrm{N}$ (see also Fig. 6). Accelerations are given in units of $\mathrm{m} / \mathrm{s} /$ day (Panels $\mathbf{a}-\mathbf{d}, \mathbf{g}$, and $\mathbf{h}$ ), relative contributions are given in percent (Panels $\mathbf{e}$ and $\mathbf{f}$ ). Again, for comparison the contour lines of the zonal mean zonal wind taken from ECMWF are shown. 

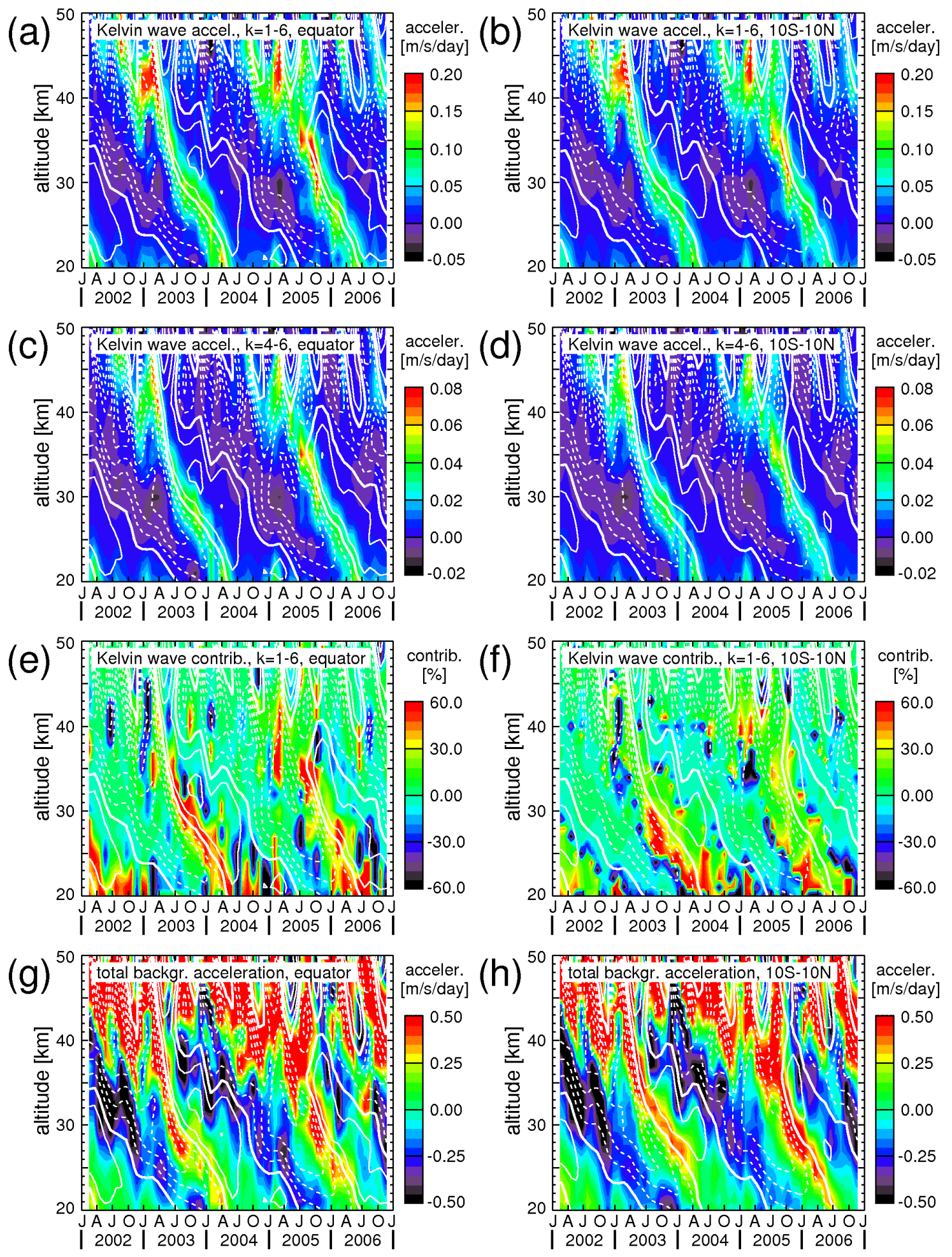

Fig. 10. Same as in Fig. 9 but for ECMWF data. 
Total Kelvin wave $(k=1-6)$ peak accelerations are observed during periods of strong westerly wind shear. For SABER values as high as about $0.15-0.20 \mathrm{~m} / \mathrm{s} /$ day are reached at the equator and somewhat lower if accelerations are averaged over the latitude band $10^{\circ} \mathrm{S}-10^{\circ} \mathrm{N}$ (see Fig. 9a and $b$ ). Accelerations due to Kelvin waves are highest when the zonal mean zonal wind is in the range between -20 and $0 \mathrm{~m} / \mathrm{s}$. But forcing by Kelvin waves is still present at the zero wind line and even extends into the phases of westerly wind as long as there is westerly wind shear. Thereby forcing by Kelvin waves contributes to the build-up of the westerly QBO wind jet.

This effect might be caused by high phase speed Kelvin waves, which do not encounter a critical wind level $\hat{c}_{\phi}=\bar{u}$ (i.e., the intrinsic phase speed $\hat{c}_{\phi}=\hat{\omega} / k$ and the zonal mean background wind $\bar{u}$ become equal), but will be subject to enhanced radiative damping. Radiative damping is inversely proportional to the vertical group velocity $c_{g z}$ of the Kelvin waves (see Shiotani and Horinouchi, 1993; Randel and Wu, 2005), and since $c_{g z} \sim\left|\bar{u}-\hat{c}_{\phi}\right|^{2}$ the damping is increased if the difference between intrinsic phase speed and zonal background wind is reduced (which is the case during westerly wind shear).

Another effect that can be found in Fig. 9a and $b$ is an annual cycle of Kelvin wave accelerations at about $20 \mathrm{~km}$ altitude. Annual variations of Kelvin wave activity have been reported before, for example, by Venkat Ratnam et al. (2006). Maximum Kelvin wave activity is always observed in Northern Hemisphere winter, which is consistent with our observations (see Figs. 3-5 and Figs. 7-10). This annual cycle is likely due to variations in the Kelvin wave source processes in the troposphere and is also reflected in annual variations found in OLR.

This annual variation of Kelvin wave accelerations with enhanced westerly acceleration during Northern Hemispheric winter seems to be quite important in the maintenance of the QBO westerly jets for an extended period at low levels, which is, for example, observed at the beginning of 2003 and at the beginning of 2005 (see Fig. 9a and b). When the westerly jets remain nearly stationary at altitudes around $20 \mathrm{~km}$, before their end, there exists an approximate balance between dissipating Kelvin waves, maintaining the westerly jet, upward advection, hindering its downward progression, and other forcing terms (other wave modes and the other terms that can be derived from the background atmosphere, see Eq. 9). For an error discussion of Kelvin wave momentum fluxes, accelerations, as well as uncertainties of the estimate of the expected total wave forcing see Sect. 5.

The relative structures of the high zonal wavenumber Kelvin wave accelerations (see Fig. 9c and d) are about the same as for the total Kelvin wave accelerations. Please note that the color scales in Fig. 9a and b are different from the ones in Fig. 9c and d. The contribution due to high zonal wavenumbers $(k=4-6)$ is about $30 \%$ of the total Kelvin wave accelerations. Accelerations due to zonal wavenumbers 3-6 (not shown) contribute even as much as $50-60 \%$ to the total Kelvin wave forcing. This means that the contribution of high zonal wavenumber Kelvin waves is too large to be neglected and studies that cover only zonal wavenumbers 1 and 2 cover only about half of the total Kelvin wave forcing of the background wind.

At the equator in the altitude range $20-35 \mathrm{~km}$ forcing by Kelvin waves alone is often sufficient to account for almost the whole total wave forcing during the later parts of periods of strong westerly wind shear, i.e. when the zonal wind is in the range between -20 and $10 \mathrm{~m} / \mathrm{s}$ (see Fig. 9e). Early in the phases of strong westerly wind shear, when the zonal mean easterly winds are still stronger (i.e., more negative) than about $-20 \mathrm{~m} / \mathrm{s}$, the contribution of Kelvin waves to the total wind forcing is small (less than about 20\%). This can be seen alone from the fact that peak values of total eastward wave forcing are over twice as high as peak values of total Kelvin wave forcing (see Fig. 9g). Also at higher altitudes (above about $40 \mathrm{~km}$ ) the contribution of the Kelvin waves is low (less than about 20-30\%).

During the seasonal peaks of Kelvin wave forcing in Northern Hemispheric winter around $20 \mathrm{~km}$ altitude, in the several months before the end of the QBO westerly wind jets, Kelvin wave accelerations can likely explain the whole expected total wave forcing or is even in the opposite direction, so that additional wave forcing in westward direction contributed by waves other than Kelvin waves is needed to explain the expected total wave forcing. This additional forcing could, for example, be provided by westward propagating global scale wave modes (e.g., equatorial Rossby waves) or westward propagating mesoscale gravity waves. Propagation conditions for those waves are favorable during QBO westerly wind phases and thus enhanced wave activity of those waves is expected.

The interpretation of wave forcing latitudinal averages is difficult because our estimate for the total wave forcing can change its sign depending on latitude so that latitudinally averaged values do not always make sense. Some information can however be obtained.

Kelvin wave forcing is most important around the equator. With increasing latitude especially accelerations due to the meridional advection term become stronger. One effect of the increasing importance of this advection term is that for $10^{\circ} \mathrm{S}-10^{\circ} \mathrm{N}$ latitudinal averages the peak accelerations of the total wave forcing are shifted towards later times during the phases of westerly wind shear (compare Fig. 9g and h). Therefore highest relative contributions of Kelvin wave forcing are now found earlier than at the equator (see Fig. 9f). If the ECMWF background winds are realistic in the whole $10^{\circ} \mathrm{S}-10^{\circ} \mathrm{N}$ latitudinal band this could indicate that during westerly wind shear forcing due to waves other than Kelvin waves is different between the equator and the outer parts of the $10^{\circ} \mathrm{S}-10^{\circ} \mathrm{N}$ latitudinal band (latitudes more than a few degrees off the equator). 
For ECMWF data total Kelvin wave peak accelerations at the equator are in the same range as for SABER (about $0.15-0.20 \mathrm{~m} / \mathrm{s} /$ day). Accelerations are also strongest during periods of strong westerly wind shear when the zonal mean zonal wind is in the range between -20 and $0 \mathrm{~m} / \mathrm{s}$ and it is supported that Kelvin wave accelerations seem to play an important role in the maintenance of the QBO westerly jets at low altitudes (see Fig. 10a and b). Also in the ECMWF data high zonal wavenumber Kelvin waves contribute considerably to the total Kelvin wave forcing (about $30 \%$ for zonal wavenumbers $k=4-6$ and about $50-60 \%$ for zonal wavenumbers $k=3-6)$. Again, the relative structures of the high zonal wavenumber Kelvin wave contribution (see Fig. 10c and d) are similar as for the total Kelvin wave accelerations.

At the equator, like for SABER, the contribution of the ECMWF total Kelvin wave forcing often is almost sufficient to explain the total wave forcing in the later parts of periods of strong westerly wind shear in the altitude range $20-35 \mathrm{~km}$ (see Fig. 10e). At higher altitudes the relative contribution of Kelvin waves is low.

During the seasonal peaks of Kelvin wave forcing in Northern Hemispheric winter around $20 \mathrm{~km}$ altitude, in the several months before the end of the QBO westerly wind jets, also in the ECMWF results Kelvin wave accelerations can likely explain the whole total expected wave forcing or are even in opposite direction. The Kelvin wave accelerations in the ECMWF data are however somewhat lower than for SABER.

If averaged over the latitude band $10^{\circ} \mathrm{S}-10^{\circ} \mathrm{N}$ the situation is similar to the one found for SABER. Due to the shift of the peak accelerations of the total wave forcing derived from the background atmosphere high relative values of Kelvin wave forcing are found earlier than at the equator (see Fig. 10f).

Both SABER and ECMWF results show that forcing by Kelvin waves plays an important role in the total wave forcing of the QBO during periods of strong westerly wind shear, especially at the equator, but also in the whole latitude band $10^{\circ} \mathrm{S}-10^{\circ} \mathrm{N}$.

In the altitude range $20-35 \mathrm{~km}$ Kelvin wave forcing alone is sometimes sufficient to explain the whole total wave forcing of the zonal mean zonal background flow. Mostly, however, additional wave forcing is required. This missing wave forcing can be quite strong. Peak values of the total wave forcing are over twice as high as peak values of Kelvin wave forcing and sometimes (especially at higher latitudes) forcing by Kelvin waves can be even less than about $20 \%$ of the total wave forcing. The missing wave forcing during the periods of westerly wind shear will most likely be contributed by gravity waves or intermediate scale waves. If our estimates for the total wave forcing derived from the ECMWF background atmosphere are still realistic at higher altitudes, Kelvin waves should not play an important role at altitudes above about $40 \mathrm{~km}$, i.e. for the forcing of the SAO.
There is an overall good agreement between SABER and ECMWF results. There is not only good correspondence in the peak accelerations, but also the relative structures of the SABER Kelvin wave accelerations agree remarkably well with the results obtained from ECMWF.

\subsubsection{Comparison with previous estimates}

Our peak zonal wind acceleration values due to Kelvin waves are about $0.15-0.20 \mathrm{~m} / \mathrm{s} /$ day at the equator and somewhat lower if averaged over the latitude band $10^{\circ} \mathrm{S}-10^{\circ} \mathrm{N}$ for both SABER and ECMWF during periods of strong westerly wind shear.

Peak values of accelerations due to Kelvin waves derived by Hitchman and Leovy (1988) from 7 months of LIMS satellite data are about $0.2-0.7 \mathrm{~m} / \mathrm{s} /$ day between about 30 and $40 \mathrm{~km}$ altitude based on daily values for zonal wavenumbers $1-3$ and the latitude range $10^{\circ} \mathrm{S}-10^{\circ} \mathrm{N}$. Peak values for monthly averages in the same altitude range are about $0.2 \mathrm{~m} / \mathrm{s} /$ day. Kelvin wave forcing was found to be about 20 $70 \%$ of the total momentum required for balancing the mean zonal flux in the stratosphere. This is in good agreement with our results, maybe somewhat higher than the ECMWF and smoothed SABER results. It should however be noted that the LIMS and the SABER retrieval algorithm are very similar and like in the unsmoothed SABER data there are also indications for oscillations in the accelerations derived from LIMS data.

Another study based on about one year of Cryogenic Limb Array Etalon Spectrophotometer (CLAES) data and zonal wavenumbers 1 and 2 in the latitude band $8^{\circ} \mathrm{S}-8^{\circ} \mathrm{N}$ was carried out by Canziani and Holton (1998). Canziani and Holton (1998) derived peak values of $0.18 \mathrm{~m} / \mathrm{s} /$ day for daily values at $10-20 \mathrm{hPa}$ (about $27.5-32.3 \mathrm{~km}$ altitude) and also at $50 \mathrm{hPa}$ (about $18.7 \mathrm{~km}$ altitude) shortly before Kelvin wave activity vanishes. On monthly average peak values for Kelvin wave forcing are more likely about $0.1 \mathrm{~m} / \mathrm{s} /$ day for wavenumbers 1 and 2 combined. The Kelvin wave forcing was found to be about $20-60 \%$ of the total momentum required for balancing the mean zonal flux. This is in good agreement with our results if we take into account that wavenumbers 1 and 2 provide only about $50 \%$ of the total Kelvin wave forcing (see above).

Also Tindall et al. (2006b) derived accelerations due to Kelvin waves from ERA-15 data for the $70-50 \mathrm{hPa}$ layer (about $16.2-18.7 \mathrm{~km}$ altitude). Their minimum and maximum values are about 0.02 and $0.06 \mathrm{~m} / \mathrm{s} /$ day, respectively. This compares well with our $10^{\circ} \mathrm{S}-10^{\circ} \mathrm{N}$ averages derived from SABER as well as ECMWF operational analyses which both vary between about 0 and $0.15 \mathrm{~m} / \mathrm{s} /$ day in the lowermost stratosphere. Our peak values are however somewhat higher. The good agreement between our SABER and our ECMWF results with strongly enhanced values during phases of strong westerly wind shear and the much weaker enhancements in the ERA-15 results could hint at improvements in the 

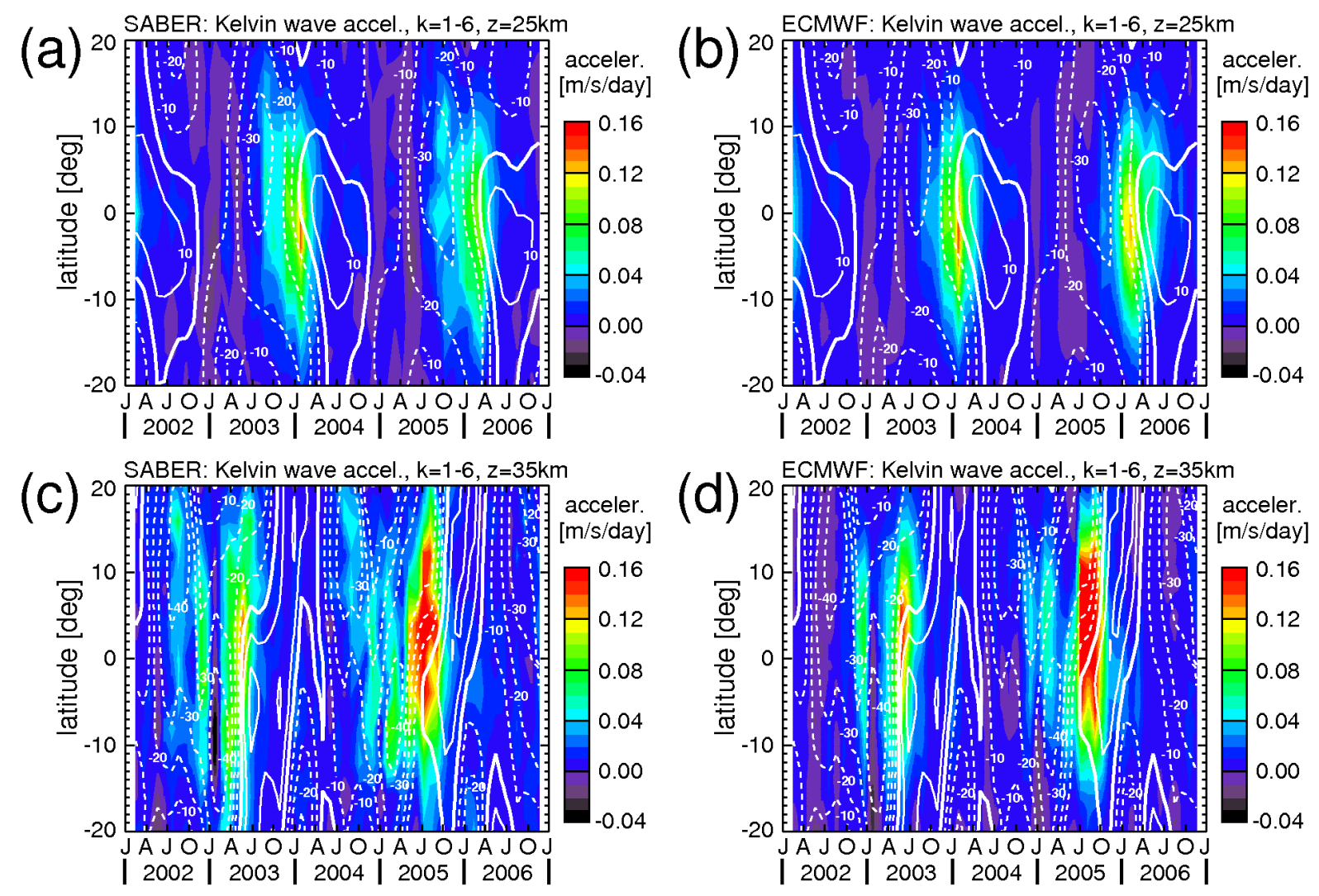

Fig. 11. Latitude-time cross sections at 25 and $35 \mathrm{~km}$ altitude for the SABER accelerations based on vertically smoothed temperature squared amplitudes (a and c) as well as for ECMWF (b and d). Values given are in units of $\mathrm{m} / \mathrm{s} / \mathrm{day}$. White contour lines indicate the zonal mean zonal wind taken from ECMWF in $\mathrm{m} / \mathrm{s}$.

ECMWF operational analyses with respect to the ERA-15 reanalyses. In ERA-15, for example, no TOVS data were used for assimilation above $100 \mathrm{hPa}$ between $20^{\circ} \mathrm{S}$ and $20^{\circ} \mathrm{N}$ and also the ECMWF model used for ERA-15 is an older version than for the operational analyses in our study.

This means that there is an overall agreement between our estimates and the previous findings by Hitchman and Leovy (1988); Canziani and Holton (1998) and Tindall et al. (2006b). Also the relative contribution of the Kelvin waves to the total eastward wave forcing is similar. Previous results cover the wide range of about $20-70 \%$ of the total wave forcing. If we take into account that in our results Kelvin wave forcing is less than about $20-30 \%$ in some parts of the periods of westerly wind shear and almost $100 \%$ in other parts, on average this is also good qualitative agreement with the previous studies.

\subsubsection{Latitude-time cross section of Kelvin wave accel- erations}

Figure 11 shows latitude-time cross sections at 25 and $35 \mathrm{~km}$ altitude for the SABER Kelvin wave accelerations based on vertically smoothed temperature squared ampli- tudes (Fig. 11a and c) as well as for ECMWF (Fig. 11b and d).

At $25 \mathrm{~km}$ altitude (Fig. 11a and b) we find the two regions of enhanced Kelvin wave accelerations which correspond to phases of strong westerly wind shear. Due to the QBO phase lag between 25 and $35 \mathrm{~km}$ these two regions show up several months earlier at $35 \mathrm{~km}$ (Fig. 11c and d). From the latitudetime cross sections we can also see that accelerations due to Kelvin waves are indeed most significant in the latitude band $10^{\circ} \mathrm{S}-10^{\circ} \mathrm{N}$ and the results are about symmetric with respect to the equator. Therefore average Kelvin wave accelerations calculated in this latitude band are representative numbers for the total contribution due to Kelvin waves. Also comparisons with model data based on this latitude band make sense because the averaged values will be more robust than values given for a single latitude.

\subsection{The importance of other wave modes for the QBO and SAO}

During the phases of QBO easterly winds Kelvin waves are by far the most dominant global scale wave mode. As shown above their contribution to the total wave forcing can 
sometimes be almost 100\% (depending on the latitudes considered) during the periods of strong westerly wind shear. Sometimes during the periods of westerly wind shear, however, large part of the total wave forcing is also missing. The missing westerly acceleration therefore has to be contributed by other waves. During periods of easterly winds there is only little activity of westward propagating equatorial wave modes like Rossby-gravity or equatorial Rossby waves (Ern et al., 2008). In addition, forcing by other eastward propagating global scale wave modes should be comparably low (Tindall et al., 2006b). This means that a considerable part of the wave forcing would have to be attributed to mesoscale gravity waves or intermediate scale waves.

During periods of easterly wind shear part of the wave forcing is provided by the westward propagating global scale Rossby-gravity or equatorial Rossby waves. But it is pointed out by Dunkerton (1997) that during easterly wind shear wave forcing by global scale waves should be even weaker than during periods of westerly wind shear. Again, a considerable part of the wave forcing would have to be provided by mesoscale gravity waves or intermediate scale waves.

The importance of those waves is in good agreement with the results by several previous studies (e.g., Dunkerton, 1997; Alexander and Rosenlof, 2003) who attribute considerable part of the QBO wave forcing to waves not resolved by measurements or the model data used (i.e., mesoscale gravity waves or intermediate scale waves).

Our results in Figs. 9 and 10 show that the contribution of global scale Kelvin waves to the SAO is almost negligible which can be seen from the very low percentages of Kelvin wave forcing at altitudes above $40 \mathrm{~km}$. It should however be noted that at those altitudes the background wind forcing derived from ECMWF data will be subject to larger errors. Not only the contribution of Kelvin waves but also contributions due to other global scale wave modes will likely be low at those altitudes. This again underlines the importance of mesoscale waves for the driving of both the QBO and, in particular, the SAO. This confirms the results of, for example, Ray et al. (1998) or Alexander and Rosenlof (2003) who attribute also large part of the forcing of the SAO to mesoscale gravity waves. For further references see also the review paper by Baldwin et al. (2001).

This demonstrates the importance of further highresolution measurements of those small-scale waves in the tropics (and also on global scale) to obtain a better understanding of the whole dynamics in the tropics and to provide better constraints for high resolution global models (e.g., Riese et al., 2005; Preusse et al., 2009).

\section{Error discussion}

\subsection{Uncertainties of Kelvin wave momentum fluxes and zonal wind accelerations}

The scientific interpretation of momentum fluxes and accelerations relies strongly on the accuracy of the derived values. We therefore give a detailed discussion of the leading error sources.

The first error source are offsets and noise, which are error sources typical for measurements. Systematic offsets in the temperature data, however, will have no effect on our results since only temperature perturbations are analyzed. Since the spectral background is subtracted from the space-time spectra before our analysis is carried out, also measurement noise will have only little effect, if we assume white noise.

The second error source are assumptions that are made when we apply the space-time spectral analysis. For example, we have to assume stationarity of the spectral components over the length of the time windows used (31 days). Probably these errors will be small compared to other error sources and they are hard to quantify and depend on the atmospheric state in each time window. Therefore this error source will not be treated in our work.

The most important error source is the accuracy of the derived space-time spectra. There are several effects that have to be taken into account:

1. spectral artifacts (leakage, aliasing) or non-Kelvin wave contributions

2. accuracy of the spectral background that has to be subtracted (this background is mainly due to non-resolved gravity waves)

3. observed vertical oscillation of spectral amplitudes (SABER retrieval artifact)

These three effects will be discussed in the following.

\subsubsection{Spectral artifacts and non-Kelvin wave contribu- tions}

Since Kelvin waves are the main spectral contribution in temperatures this will only be a minor effect for the low zonal wavenumbers where the bulk of Kelvin wave temperature variances is located. For higher zonal wavenumbers this can also be a considerable error source, in particular if the wavebands for integrating the spectral contributions are extended too far towards higher frequencies. This is one reason why we limited our study to frequencies lower than 0.4 cycles/day. Of course, some of the forcing will likely be missed, but the values given for frequencies lower than 0.4 cycles/day will be more reliable. For these low frequencies uncertainties due to this error source will then likely be less than $10 \%$, taking into account the very dominant spectral feature of Kelvin waves (see Figs. 1 and 2). 


\subsubsection{Accuracy of the spectral background}

Different from previous analyses (e.g., Hitchman and Leovy, 1988; Canziani and Holton, 1998) we account for the spectral background due to mesoscale gravity waves in the SABER and ECMWF space-time spectra. This spectral background is estimated from zonal wavenumbers $\geq 3$ and then subtracted to obtain the true Kelvin wave signal without bias. It is assumed that the background is constant over the whole spectral domain (white noise). Some minor deviations from this assumption can be seen in the results shown in Ern et al. (2008). In Ern et al. (2008) the spectral background is estimated from the complete (symmetric + antisymmetric) spectra as well as from the "westward" part of the antisymmetric spectra only. The results are very similar, but the estimates for the antisymmetric westward spectra are lower than the estimates for the "full" spectra: about 25\% lower for SABER and about 35\% lower for ECMWF. Based on the assumption that these values represent realistic error ranges for the spectral background, we performed simulations with the spectral background both increased and reduced by these numbers to estimate uncertainties due to this error source for both SABER and ECMWF.

For both SABER and ECMWF the effect of increasing and reducing the spectral background is lower than about one color level in Figs. 4, 5 and Figs. 7-10 for the total Kelvin wave momentum fluxes and accelerations (zonal wavenumber range $k=1-6)$. This means that in the regions with significant Kelvin wave momentum fluxes or accelerations this effect is lower than about $10 \%$. In these cases Kelvin wave variances are still considerably higher than the spectral background and minor errors of the spectral background do not play an important role.

For ECMWF this is also the case for the high zonal wavenumbers $(k=4-6)$ because the ECMWF spectral background is very low, even if compared with Kelvin wave variances at higher zonal wavenumbers.

For the SABER high zonal wavenumbers $(k=4-6)$ the accuracy of the spectral background plays a somewhat more important role because the spectral background is higher than for ECMWF. However, the variation of Kelvin wave momentum fluxes and accelerations is still about one color level for both momentum fluxes and accelerations. This corresponds to estimated errors of about $0.005 \mathrm{mPa}$ and $0.005 \mathrm{~m} / \mathrm{s} / \mathrm{day}$, respectively.

\subsubsection{Vertical oscillation of SABER temperature vari- ances}

As already discussed in Sect. 4.2.1 there is an artificial oscillation in the SABER Kelvin wave momentum fluxes. This effect is amplified when calculating vertical gradients in order to determine acceleration values (see Fig. 7). Therefore SABER temperature variances are smoothed vertically to remove this oscillation (see Fig. 9). Since for the SABER ac- celerations the effect of the oscillation looks very similar for the two zonal wavenumber ranges $k=1-6$ and $k=4-6$, respectively, this error likely has the characteristics of a relative error. The comparison between SABER and ECMWF results suggests that smoothing the SABER data vertically with a suitable length of about $4 \mathrm{~km}$ leads to an almost complete cancellation of this artifact.

A sinusoidal oscillation in temperature variance vertical profiles will also result in a sinusoidal oscillation in the derived wave accelerations. (The derivative of a sinusoid is a sinusoid of the same wavelength.) Also for a weakly damped oscillation (like in our case) an error discussion based on accelerations is about equivalent to an error discussion based on temperature variances. Therefore we will perform the error analysis directly for the accelerations, where the oscillations can be seen more clearly, and where this error has the largest effect. In addition, the error discussion directly for accelerations can be performed more easily.

Errors that are still remaining in the smoothed data (Fig. 9) are estimated in the following way: In a simulation we mimic the vertical variations of the accelerations that are observed in Figs. 7 and 9. We assume a perturbation with the characteristics of a harmonic oscillation of 4-6 km vertical wavelength superimposed on the "undistorted" Kelvin wave accelerations. The undistorted accelerations are assumed as a peak of 5-7 km vertical extent and triangle or quadratic shape as function of altitude. The perturbation (oscillation) is assumed to have an initial amplitude the same size as the assumed "undistorted" acceleration peak, which is a very conservative assumption. Just like the oscillation observed in Fig. 7 the simulated oscillation is damped to zero towards lower altitudes over an altitude interval of about $10 \mathrm{~km}$.

This situation is illustrated in Fig. 12 for the typical case of a $7 \mathrm{~km}$ wide triangular peak for the undistorted accelerations (green dotted) with a maximum value of $0.15 \mathrm{~m} / \mathrm{s} /$ day at $31 \mathrm{~km}$ altitude. On the undistorted accelerations we superimpose a strong oscillation of $5 \mathrm{~km}$ vertical wavelength (black dotted) and an initial amplitude of $0.15 \mathrm{~m} / \mathrm{s} / \mathrm{day}$, which is damped to zero towards lower altitudes over an altitude range of $10 \mathrm{~km}$. The resulting signal that would be observed is given by the red solid line. Like in Fig. 7 there are two negative acceleration "dips" which are caused by the perturbation. I.e., the two bands of negative accelerations that can be seen in Fig. 7 can be explained by an overshooting of the perturbation (oscillation) towards negative values. The blue solid line is obtained after smoothing the red line vertically by a 4-km running mean, corresponding to the smoothed SABER results shown in Fig. 9. The light blue dotted line, which represents the deviation between the green dotted line of the assumed "true" peak and the smoothed data (blue line) can then be taken as the expected error of the smoothed data.

The simulations made here are very simplified, but are able to reproduce the observed features. Though some dependence on the assumptions made here exists, this raises confidence in the resulting error estimates. However, the 


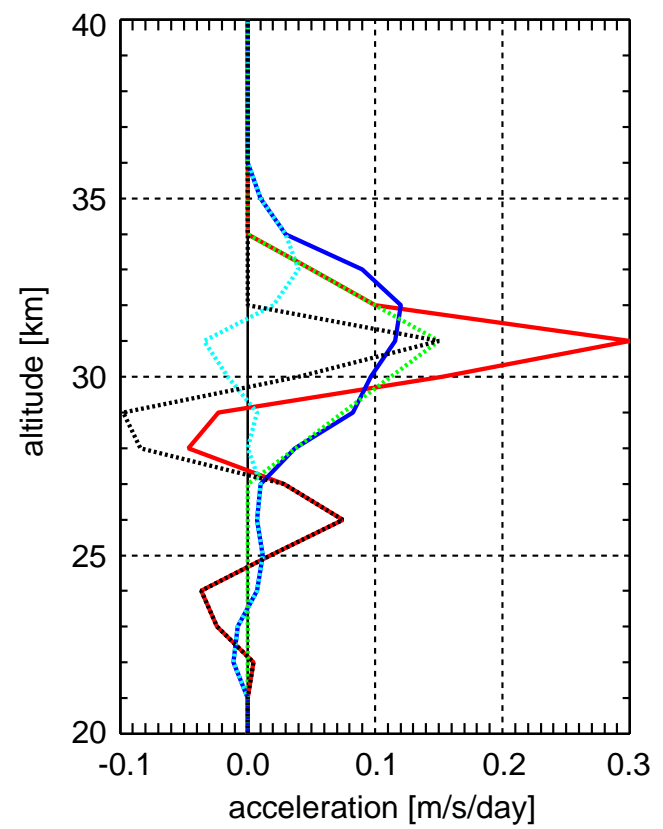

Fig. 12. Illustration of the error estimation for Kelvin wave zonal wind accelerations. A sinusoidal perturbation (black dotted) is superimposed on the assumed true acceleration peak (green dotted). The resulting signal that is observed is given by the red solid line (cf. results shown in Fig. 7). The blue solid line is the observed signal smoothed vertically by a 4-km running mean (cf. results shown in Fig. 9). The light blue dotted line is the deviation of the smoothed signal from the assumed true signal and can be taken as an estimate for the expected error. For details see text.

characteristics of the perturbation at altitudes above the location of the acceleration maximum is very hard to quantify without carrying out dedicated retrieval simulations.

For zonal wavenumbers $k=1-6$ (total acceleration) the results of our error estimation show that the integral over the whole acceleration peak has an error of about $20 \%$. For the peak value itself the error is about $30 \%$ or somewhat lower or higher, depending on assumptions. At altitudes somewhat below the acceleration maximum the error should be less than about $0.02 \mathrm{~m} / \mathrm{s} /$ day.

Of course, due to the vertical smoothing there is some vertical smearing of the acceleration peak. Therefore the peak value will be low-biased. On the other hand, the integral over the acceleration peak will likely be high-biased, and there will also be likely a high-bias at the upper edge of the acceleration peak. However, as mentioned above, the characteristics of the perturbation at altitudes above the location of the acceleration maximum is not as well characterized as at lower altitudes.

Momentum fluxes can be obtained by integrating vertically over the accelerations (see Eq. 8). Adopting relative errors estimated for the accelerations also for the momentum fluxes would mean that no cancellation effects are assumed
Table 1. Error estimates for SABER and ECMWF Kelvin wave momentum fluxes and accelerations for the zonal wavenumber $k=1-$ 6 wave band and different cases.

\begin{tabular}{lc}
\hline case & SABER and ECMWF \\
\hline momentum flux, & $\max (\sim 10 \%, 0.015 \mathrm{mPa})$ \\
weak vertical gradient & $\sim 30 \%$ \\
momentum flux, & \\
strong vertical gradient & $\sim 30 \%$ \\
\hline acceleration, peak value & $\sim 20 \%$ \\
acceleration, integral over peak & $0.02 \mathrm{~m} / \mathrm{s} /$ day \\
acceleration, outside peak & \\
\hline
\end{tabular}

for this integration. Relative errors of the accelerations can therefore be taken as an upper limit for the error of momentum fluxes. In this way we obtain an error estimate of about $30 \%$ for the momentum fluxes when strong vertical gradients are present.

If there are only small vertical gradients the error of momentum flux due to the oscillation artifact should be negligible after smoothing the SABER data. This can be seen from the very small acceleration error values outside the acceleration peak (see Fig. 12). The error of momentum flux will then be dominated by the accuracy of the spectral background (see Sect. 5.1.2).

\subsubsection{Summary of error ranges}

For both SABER and ECMWF the accuracy of the $k=1-6$ momentum fluxes is in the $10 \%$ range if no vertical gradients are present. For very low momentum fluxes the error will be somewhat larger, at least $0.015 \mathrm{mPa}$ (corresponding to one color level in Figs. 4, 5, and 8). Where strong vertical gradients are present, for SABER the error is in the range of about 30\%. For ECMWF the error in the case of vertical gradients is difficult to estimate. There is no vertical oscillation like in the SABER data, on the other hand there will be error sources that are not easily accessible to us (for example, model assumptions). Therefore we assume like for SABER an error of about $30 \%$.

For SABER the accuracy of the $k=1-6$ accelerations is about $30 \%$ (likely a low-bias) for the peak values during strong westerly wind shear. The integral over the acceleration peak is somewhat more accurate and has an error of about 20\% (likely a high-bias). Outside the acceleration peak the error of the accelerations is about $0.02 \mathrm{~m} / \mathrm{s} /$ day. Like for the momentum fluxes we assume the same error ranges for the ECMWF data. The error ranges are summarized in Table 1 .

In regions of strong vertical momentum flux gradients error ranges for the high zonal wavenumbers $k=4-6$ in smoothed SABER results should be about the same as for 
the zonal wavenumber range $k=1-6$ (for both accelerations and momentum fluxes), taking into account that errors due to the artificial oscillation are the dominant effect. Relative errors for regions with only weak vertical gradients might be somewhat (but not much) larger than the values given in Table 1. Minimum errors for zonal wavenumbers $k=4-6$ are about $0.005 \mathrm{mPa}$ and $0.005 \mathrm{~m} / \mathrm{s} /$ day for momentum fluxes and accelerations, respectively.

There are also some weaker vertical increases of momentum flux visible in ECMWF data and also in the vertically smoothed SABER data. Those increases cannot be explained easily. Possibly this shows the limitations of our method and can also be taken as a measure for the expected errors of momentum flux, in addition to the values given in Table 1. It should however be noted that the vertical gradients introduced by these momentum flux increases are rather weak and do not affect the accelerations due to Kelvin waves derived during periods of strong westerly wind shear.

\subsection{Uncertainties of the acceleration terms derived from an ECMWF background atmosphere}

\subsubsection{Uncertainties of ECMWF zonal mean meridional and vertical winds}

As already stated in Sect. 4.1 the zonal mean meridional and vertical winds taken from ECMWF are highly uncertain. This uncertainty enters the meridional and vertical advection terms of the background atmosphere contributing to the estimate for the expected total forcing of the zonal wind by waves (see Fig. 6). Therefore also the values given for the relative contribution of the zonal wind forcing due to Kelvin waves will be affected.

To give approximate error ranges for the relative contribution of the Kelvin waves we perform a sensitivity study with the zonal mean meridional and vertical winds both reduced and increased by 50\%. The results are given in Fig. 13 for the equator. The left column in Fig. 13 shows the different cases of increased and reduced meridional and vertical wind velocity. The right column shows the relative contribution of the Kelvin waves for the respective case in the left column. Relative contributions are given for the vertically smoothed SABER data only, because ECMWF relative contributions are similar.

A reduction (Fig. 13a) or an increase (Fig. 13c) of the zonal mean meridional wind velocity by $50 \%$ has only some minor effects at altitudes below about $35 \mathrm{~km}$. This is the case because in this altitude range at the equator meridional gradients of the zonal wind are low and thus also accelerations due to the meridional advection term are low. At altitudes above $35 \mathrm{~km}$, however, we can see that the estimate for the relative contribution of Kelvin waves to the total wave forcing (see Fig. 13b and d) becomes highly uncertain.

A reduction (Fig. 13e) or an increase (Fig. 13g) of the zonal mean vertical wind velocity by $50 \%$ has only some mi- nor effects on the estimate for the Kelvin wave relative contribution during the periods of strong westerly wind shear (see Fig. 13f and h). This is the case because the vertical advection term derived from the ECMWF background atmosphere is comparably low during the periods of strong Kelvin wave forcing (see Fig. 6).

For the seasonal peaks of Kelvin wave forcing in Northern Hemispheric winter around $20 \mathrm{~km}$ altitude the variation of meridional and vertical winds has only little effect for the findings in Sect. 4.2.2. Close to the end of the QBO westerly wind phases, for all cases shown, Kelvin wave accelerations are able to account for the whole expected total wave forcing or are even in opposite direction, so that easterly wave forcing by waves other than Kelvin waves is needed to explain the total wave forcing.

For averages over the latitude band $10^{\circ} \mathrm{S}-10^{\circ} \mathrm{N}$ the influence of the assumed error ranges for the meridional and vertical winds is similar to the effects shown in Fig. 13 for the equator. Therefore the results of the sensitivity study for the $10^{\circ} \mathrm{S}-10^{\circ} \mathrm{N}$ averages are not shown here.

It should however be noted that uncertainties induced by the ECMWF mean meridional and vertical winds will only affect our estimates for the relative contribution of Kelvin wave accelerations to the expected total forcing by atmospheric waves, but not the Kelvin wave accelerations itself.

\subsubsection{Choice of the window length for the space-time spectral analysis}

The chosen length of the time windows for our spectral analysis is a tradeoff between temporal and spectral resolution. Especially at the lower altitudes there is significant spectral power at wave periods as long as about 15 days, but somewhat less for wave periods that are even longer. This can be seen, for example, from the values given for the strongest wave components in average Kelvin wave spectra, which are summarized in Table 1 in Ern et al. (2008). These findings indicate that the chosen length of the time windows of 31 days is sufficient to properly resolve most of the Kelvin waves even at lower altitudes. On the other hand the existence of significant spectral power at wave periods as long as about 15 days at low altitudes also indicates that the length of the time windows cannot be significantly reduced.

The question arises whether the length of the time windows (31 days) used in our spectral analysis might affect the vertical gradients of the zonal wind entering the vertical advection term in Eq. (6). Of course, additional smoothing of the background atmosphere is introduced by the 31-day averages of the background atmosphere. The zonal wind can change as much as about $20 \mathrm{~m} / \mathrm{s}$ during one 31-day period. This is similar to the changes of the zonal wind that can occur vertically from one ECMWF pressure level to the next. The size of the effect caused by the 31-day averages can therefore be estimated from the vertical variations. 

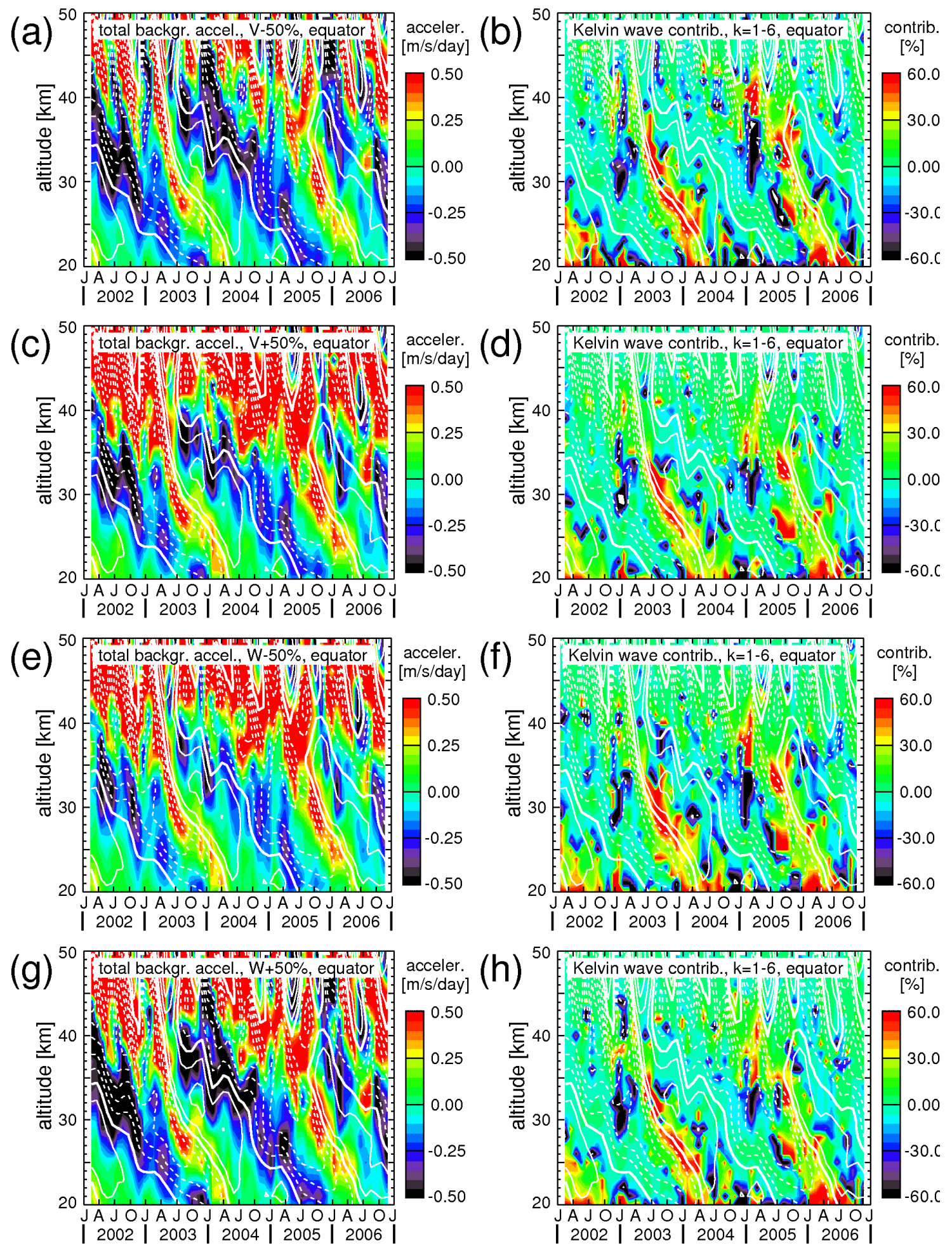

Fig. 13. Estimates of the expected total acceleration due to atmospheric waves derived from an ECMWF background atmosphere at the equator for the cases: average meridional wind reduced (a) as well as increased (c) by 50\% and also for the average vertical wind reduced (e) as well as increased (g) by 50\%. In the right column also the relative contribution (for vertically smoothed SABER data only) of Kelvin waves to the total wave forcing is given for the respective case in the left column. Accelerations (left column) are given in units of $\mathrm{m} / \mathrm{s} / \mathrm{day}$, relative contributions (right column) are given in percent. Again, for comparison the contour lines of the zonal mean zonal wind taken from ECMWF are shown. 
In vertical direction we applied both linear and quadratic interpolation of the background atmosphere, but the results did not change significantly. Changes are within about one color level in Figures 6, 7 and 9-11. This indicates that the smoothing of both horizontal and vertical variations of the background atmosphere, which is inevitable during our analysis, is only a minor effect for the results presented.

\section{Summary and conclusions}

In our study we derive spectra of the vertical flux of horizontal momentum for equatorial global scale Kelvin waves from temperature zonal wavenumber/frequency spectra. In order to obtain the temperature spectra we carried out a windowed longitude-time spectral analysis with a series of non-overlapping 31-day time windows for both SABER and ECMWF data in the period 1/2002 until 11/2006. From the space-time spectra also altitude-time cross sections are derived.

As expected from previous studies zonal wavenumbers higher than 3 also become important, if momentum flux or accelerations are considered instead of temperatures. A comparison of SABER and ECMWF momentum flux spectra however gives us confidence that the sampling pattern of satellites in low Earth orbit (which can resolve only zonal wavenumbers up to 6-7 unambiguously) is sufficient to quantify momentum flux without major aliasing of high zonal wavenumber contributions. Momentum flux values are about $0.3 \mathrm{mPa}$ in the lower stratosphere and decrease gradually with altitude when the Kelvin waves dissipate.

Based on the derived momentum fluxes the acceleration of the zonal mean zonal wind due to Kelvin waves can be estimated. The SABER accelerations show some oscillations which are possibly a retrieval artifact. These oscillations are removed by smoothing the squared temperature amplitudes vertically by a $4-\mathrm{km}$ running mean. Peak values of accelerations for both SABER and ECMWF are about 0.15$0.20 \mathrm{~m} / \mathrm{s} /$ day during phases of strong westerly wind shear at the equator and somewhat lower if values are averaged over the latitude band $10^{\circ} \mathrm{S}-10^{\circ} \mathrm{N}$. These values are in good agreement with previous findings.

Zonal wind forcing due to Kelvin waves is sufficient to explain almost the whole total wave forcing in the later parts of the periods of strong westerly wind shear at the equator. During other parts of westerly wind shear periods, however, Kelvin waves play only a minor role, and also towards higher latitudes the importance of Kelvin waves decreases. Large part of the forcing of the QBO is therefore attributed to mesoscale gravity waves or intermediate scale waves. For $10^{\circ} \mathrm{S}-10^{\circ} \mathrm{N}$ meridional averages high relative contributions due to Kelvin waves are somewhat shifted in time compared with the equator.

It is also found that seasonal variations of Kelvin wave activity, and thus Kelvin wave accelerations, could play an im- portant role for the maintenance of the QBO westerly wind jets for an extended period in the lower stratosphere. In the upper stratosphere forcing of the SAO due to Kelvin waves is only a minor effect. It is also shown that forcing due to equatorial Kelvin waves is mainly confined to the latitude band $10^{\circ} \mathrm{S}-10^{\circ} \mathrm{N}$. Therefore average values for this latitude band can serve as a robust reference for comparison with GCM results.

\section{Appendix A}

\section{Kelvin wave momentum flux expressed in terms of temperature perturbations}

The forcing $F_{\mathrm{KW}}$ exerted by Kelvin waves is given by the vertical gradient of the flux of zonal momentum due to Kelvin waves alone:

$F_{\mathrm{KW}}=\frac{\partial}{\partial z}\left(\overline{u_{\mathrm{KW}}^{\prime} w_{\mathrm{KW}}^{\prime}}\right)$

with $u_{\mathrm{KW}}^{\prime}$ and $w_{\mathrm{KW}}^{\prime}$ the zonal and vertical wind perturbations due to Kelvin waves (see also Eq. (8)). For convenience we will drop the subscript "KW" in the following. From now on perturbations of the zonal mean will always be considered to be due to Kelvin waves.

If the time-averaging denoted by the overbar is carried out for the term $\overline{\left(\overline{u^{\prime} w^{\prime}}\right)}$, the vertical flux of horizontal wave momentum $\mathcal{F}_{p x}$ can be written in terms of the complex wave amplitudes $\widetilde{u}$ and $\widetilde{w}$ (similar as in Eq. (A4) in Ern et al. (2004) which is given for a linear wave approach like in Eq. (14) in Fritts and Alexander, 2003):

$\mathcal{F}_{p x}=\frac{\bar{\varrho}}{2} \operatorname{Re}\left\{\widetilde{u} \widetilde{w}^{*} e^{z / H}\right\}$

with $\widetilde{w}^{*}$ being the complex conjugate of $\widetilde{w}$. The altitude dependence $e^{z / H}$ cancels out with the altitude dependence of the atmospheric density $\bar{\varrho}$. And in the following we will always use amplitudes as well as the atmospheric density $\bar{\varrho}_{0}$ at a given reference altitude.

In the following we want to replace the wind amplitudes, first $\widetilde{w}$ and in a second step also $\widetilde{u}$, to express the Kelvin wave momentum flux in terms of temperature amplitudes. We use the polarization relations given in Fritts and Alexander (2003) and Ern et al. (2004), which are valid for a general linear wave approach.

We start from Eqs. (15) and (18) in Fritts and Alexander (2003):

$$
\begin{aligned}
& -i \hat{\omega} \tilde{u}-f \widetilde{v}+i k \tilde{p}=0 \\
& -i \hat{\omega} \widetilde{\Theta}+\left(N^{2} / g\right) \widetilde{w}=0
\end{aligned}
$$

Here $\hat{\omega}$ is the intrinsic frequency, $\widetilde{u}, \widetilde{v}$ and $\widetilde{w}$ are the zonal, meridional and vertical wind amplitudes of the wave, $k$ the zonal wavenumber, $\widetilde{p}=\widehat{p} / \bar{\rho}$ the pressure amplitude $\widehat{p}$ divided 
by the atmospheric background density $\bar{\rho}, \widetilde{\Theta}$ the relative potential temperature amplitude $(\widehat{\Theta} / \Theta), N$ the buoyancy frequency, $f$ the Coriolis frequency, and $g$ the gravity acceleration.

In addition we make use of Eq. (A7) in Ern et al. (2004):

$\widetilde{w}=\frac{-\hat{\omega}}{N^{2}-\hat{\omega}^{2}}\left(m+i\left(\frac{1}{2 H}-\frac{g}{c_{s}^{2}}\right)\right) \widetilde{p}$

Here $m$ is the vertical wavenumber, $H$ the pressure scale height, and $c_{s}$ the sound speed.

For Kelvin waves the meridional wind amplitude $\widetilde{v}$ in Eq. (A3) is zero. By combining Eqs. (A3), (A4) and (A5), replacing $\widetilde{w}$ and $\widetilde{p}$, we obtain the polarization relation between $\widetilde{\Theta}$ and $\widetilde{u}$, which can be written as follows:

$\widetilde{\Theta}=\frac{i}{g} \frac{1}{1-\hat{\omega}^{2} / N^{2}}\left[m+i\left(\frac{1}{2 H}-\frac{g}{c_{s}^{2}}\right)\right] \frac{\hat{\omega}}{k} \widetilde{u}$

Since for Kelvin waves $\hat{\omega}^{2} \ll N^{2}$, this equation can be further simplified:

$\widetilde{\Theta}=\frac{i}{g}\left[m+i\left(\frac{1}{2 H}-\frac{g}{c_{s}^{2}}\right)\right] \frac{\hat{\omega}}{k} \widetilde{u}$

We further assume that all motions are adiabatic. The potential temperature is defined as follows:

$\Theta=T\left(p_{0} / p\right)^{\kappa}$

Here $p_{0}$ is the reference (surface) pressure and $\kappa=1-1 / \gamma$ with $\gamma$ the adiabatic coefficient:

$\gamma=c_{p} / c_{v}$

which is the ratio of the specific heats for constant pressure and constant volume. For diatomic gases like air: $\gamma=1.4$.

From Eq. (A8) we obtain the relationship between relative potential temperature amplitude $\widetilde{\Theta}$, relative temperature amplitude $\widetilde{T}=\widehat{T} / T$ and the pressure amplitude $\widetilde{p}=\widehat{p} / \bar{\rho}$ :

$\widetilde{\Theta}=\widetilde{T}-\frac{\gamma-1}{c_{s}^{2}} \tilde{p}$

and together with Eq. (A3), assuming $\widetilde{v}=0$ for Kelvin waves, $\tilde{p}$ can be eliminated:

$\widetilde{\Theta}=\widetilde{T}-\frac{\gamma-1}{c_{s}^{2}} \frac{\hat{\omega}}{k} \widetilde{u}$

By combining Eqs. (A11) and (A7) we obtain the polarization relation between temperature perturbation and zonal wind perturbation:

$\widetilde{T}=\left[\frac{\gamma}{c_{s}^{2}}-\frac{1}{2 g H}+\frac{i}{g} m\right] \frac{\hat{\omega}}{k} \widetilde{u}$

Or formulated for zonal wind perturbations:

$\tilde{u}=\frac{k}{\hat{\omega}} \widetilde{T} /\left[\frac{\gamma}{c_{s}^{2}}-\frac{1}{2 g H}+\frac{i}{g} m\right]$ and consequently, for absolute values:

$|\widetilde{u}|=\frac{k}{\hat{\omega}}|\widetilde{T}| / \sqrt{\left(\frac{\gamma}{c_{s}^{2}}-\frac{1}{2 g H}\right)^{2}+\frac{m^{2}}{g^{2}}}$

For Kelvin waves the perturbation $\widetilde{w}$ can be obtained by combining Eqs. (A4) and (A7), yielding:

$\widetilde{w}=\left[-m-i\left(\frac{1}{2 H}-\frac{g}{c_{s}^{2}}\right)\right] \frac{1}{k} \frac{\hat{\omega}^{2}}{N^{2}} \widetilde{u}$

Combining Eqs. (A2) and (A15) we obtain for the Kelvin wave momentum flux:

$$
\begin{aligned}
\mathcal{F}_{p x} & =\frac{\bar{\varrho}_{0}}{2} \operatorname{Re}\left\{\left[-m+i\left(\frac{1}{2 H}-\frac{g}{c_{s}^{2}}\right)\right] \frac{1}{k} \frac{\hat{\omega}^{2}}{N^{2}} \widetilde{u} \widetilde{u}^{*}\right\} \\
& =-\frac{1}{2} \bar{\varrho}_{0} \frac{m}{k} \frac{\hat{\omega}^{2}}{N^{2}}|\widetilde{u}|^{2}
\end{aligned}
$$

with the same convention as in Fritts and Alexander (2003) that negative $m$ means positive upward flux.

The absolute value $|\widetilde{u}|$ can be obtained from Eq. (A14) to use temperature fluctuations instead of zonal wind fluctuations. We finally obtain:

$\mathcal{F}_{p x}=-\frac{\bar{\varrho}_{0}}{2} \frac{k m}{N^{2}}|\widetilde{T}|^{2} /\left[\left(\frac{\gamma}{c_{s}^{2}}-\frac{1}{2 g H}\right)^{2}+\frac{m^{2}}{g^{2}}\right]$

This equation can be rewritten, replacing $m$ via the dispersion relation for Kelvin waves $\hat{\omega} / k=-N / m$ :

$\mathcal{F}_{p x}=\frac{\bar{\varrho}_{0}}{2} \frac{k^{2}}{N \hat{\omega}}|\widetilde{T}|^{2} /\left[\left(\frac{\gamma}{c_{s}^{2}}-\frac{1}{2 g H}\right)^{2}+\left(\frac{N k}{g \hat{\omega}}\right)^{2}\right]$

with $|\widetilde{T}|=\widehat{T} / T$, the temperature amplitude $\widehat{T}$ divided by the atmospheric background temperature $T$. Equation (A18) will be used to obtain momentum flux spectra for Kelvin waves from temperature spectra.

For short vertical wavelengths in Eq. (A17) the first squared term in the bracket can be neglected, and we obtain the following simple equation:

$\mathcal{F}_{p x}=-\frac{1}{2} \bar{\varrho}_{0} \frac{k}{m}\left(\frac{g}{N}\right)^{2}|\widetilde{T}|^{2}$

This approximation is valid for vertical wavelengths shorter than about $30 \mathrm{~km}$. But in our studies we will use the full equation (Eq. A18) because also longer vertical wavelengths will be considered. The approximation shown in Eq. (A19) is also known from Ern et al. (2004) for gravity waves.

Acknowledgements. The work of M. Ern was supported by the European Commission (European Union's 6th framework program) within the EC Integrated Project SCOUT-O3 (505390GOCE-CT-2004). We thank the whole SABER team, in particular M. G. Mlynczak, J. M. Russell III, and L. L. Gordley for providing the excellent data set of SABER temperatures. Thanks also go to the European Centre for Medium-Range Weather Forecasts (ECMWF) for providing the ECMWF operational analyses used. Helpful comments by two anonymous reviewers are acknowledged.

Edited by: W. Lahoz 


\section{References}

Alexander, M. J. and Rosenlof, K. H.: Gravity-wave forcing in the stratosphere: Observational constraints from the Upper Atmosphere Research Satellite and implications for parameterization in global models, J. Geophys. Res., 108, 4587, doi:10.1029/2003JD003373, 2003.

Alexander, S. P., Tsuda, T., Kawatani, Y., and Takahashi, M.: Global distribution of atmospheric waves in the equatorial upper troposphere and lower stratosphere: COSMIC observations of wave mean flow interactions, J. Geophys. Res., D24115, doi:10.1029/2008JD010039, 2008.

Andrews, D. G. and McIntyre, M. E.: Planetary waves in horizontal and vertical shear: The generalized Eliassen-Palm relation and the mean zonal acceleration, J. Atmos. Sci., 33, 2031-2048, 1976.

Angell, J. K. and Korshover J.: Evidence for a quasi-biennial variation in eccentricity of the North Polar vortex, J. Atmos. Sci., 32, 634-635, 1975.

Baldwin, M. P. and Dunkerton, T. J.: Quasi-biennial modulations of the Southern Hemisphere stratospheric polar vortex, Geophys. Res. Lett., 25, 3343-3346, 1998.

Baldwin, M. P. and Dunkerton, T. J.: Propagation of the Arctic Oscillation from the stratosphere to the troposphere, J. Geophys. Res., 104, 30937-30946, 1999.

Baldwin, M. P., Gray, L. J., Dunkerton, T. J., Hamilton, K., Haynes, P. H., Randel, W. J., Holton, J. R., Alexander, M. J., Hirota, I., Horinouchi, T., Jones, D. B. A., Kinnersley, J. S., Marquardt, C., Sato, K., and Takahashi, M.: The quasi-biennial oscillation, Rev. Geophys., 39, 179-229, 2001.

Bergman, J. W. and Salby, M. L.: Equatorial wave activity derived from fluctuations in observed convection, J. Atmos. Sci., 51, 3791-3806, 1994.

Campbell, L. J. and Shepherd, T. G.: Constraints on wave drag parameterization schemes for simulating the Quasi-Biennial Oscillation. Part I: Gravity wave forcing, J. Atmos. Sci., 62, 41784195, 2005a.

Campbell, L. J. and Shepherd, T. G.: Constraints on wave drag parameterization schemes for simulating the Quasi-Biennial Oscillation. Part II: Combined effects of gravity waves and equatorial planetary waves, J. Atmos. Sci., 62, 4196-4205, 2005 b.

Canziani, P. O. and Holton, J. R.: Kelvin waves and the quasibiennial oscillation: An observational analysis, J. Geophys. Res., 103, 31509-31521, 1998.

Dunkerton, T. J.: The role of gravity waves in the quasi-biennial oscillation, J. Geophys. Res., 102, 26053-26076, 1997.

ECMWF: Atmospheric model identification numbers: http://www. ecmwf.int/products/data/technical/model_id/, last access: 11 May 2009.

Eguchi, N. and Shiotani, M.: Intraseasonal variations of water vapor and cirrus clouds in the tropical upper troposphere, J. Geophys. Res., 109, D12106, doi:10.1029/2003JD004314, 2004.

Ern, M., Preusse, P., Alexander, M. J., and Warner, C. D.: Absolute values of gravity wave momentum flux derived from satellite data, J. Geophys. Res., 109, D20103, doi:10.1029/2004JD004752, 2004.

Ern, M., Preusse, P., and Warner, C. D.: Some experimental constraints for spectral parameters used in the Warner and McIntyre gravity wave parameterization scheme, Atmos. Chem. Phys., 6, 4361-4381, 2006, http://www.atmos-chem-phys.net/6/4361/2006/.

Ern, M., Preusse, P., Krebsbach, M., Mlynczak, M. G., and Russell III, J. M.: Equatorial wave analysis from SABER and ECMWF temperatures, Atmos. Chem. Phys., 8, 845-869, 2008, http://www.atmos-chem-phys.net/8/845/2008/.

Ern, M., Lehmann, C., Kaufmann, M., and Riese, M.: Spectral wave analysis at the mesopause from SCIAMACHY airglow data compared to SABER temperature spectra, Ann. Geophys., 27, 407-416, 2009, http://www.ann-geophys.net/27/407/2009/.

Fritts, D. C. and Alexander, M. J.: Gravity wave dynamics and effects in the middle atmosphere, Rev. Geophys., 41(1), 1003, doi:10.1029/2001RG000106, 2003.

Fujiwara, M., Kita, K., and Ogawa, T.: Stratosphere-troposphere exchange of ozone associated with the equatorial Kelvin wave as observed with ozonesondes and rawinsondes, J. Geophys. Res., 103(D15), 19173-19182, 1998.

Fujiwara, M. and Takahashi, M.: Role of the equatorial Kelvin wave in stratosphere-troposphere exchange in a general circulation model, J. Geophys. Res., 106(D19), 22763-22780, 2001.

Fujiwara, M., Hasebe, F., Shiotani, M., Nishi, N., Vömel, H., and Oltmans, S. J.: Water vapor control at the tropopause by equatorial Kelvin waves observed over the Galápagos, Geophys. Res. Lett., 28, 3143-3146, 2001.

Garcia, R. R. and Salby, M. L.: Transient response to localized episodic heating in the tropics. Part II: Far-field behavior, J. Atmos. Sci., 44, 499-530, 1987.

Garcia, R. R., Lieberman, R., Russell III, J. M., and Mlynczak, M. G.: Large-scale waves in the mesosphere and lower thermosphere observed by SABER, J. Atmos. Sci., 62, 4384-4399, 2005.

Giorgetta, M. A., Manzini, E., and Roeckner, E.: Forcing of the quasi-biennial oscillation from a broad spectrum of atmospheric waves, Geophys. Res. Lett., 29, 1245, doi:10.1029/2002GL014756, 2002.

Giorgetta, M. A., Manzini, E., Roeckner, E., Esch, M., and Bengtsson, L.: Climatology and forcing of the quasi-biennial oscillation in the MAECHAM5 model, J. Climate, 19, 3863-3881, 2006.

Hamilton, K.: High resolution global modeling of the atmospheric circulation, Adv. Atmos. Sci., 23, 842-856, doi:10.1007/s00376006-842-3, 2006.

Hamilton, K., Takahashi, Y. O., and Ohfuchi, W.: Mesoscale spectrum of atmospheric motions investigated in a very fine resolution global general circulation model, J. Geophys. Res., 113, D18110, doi:10.1029/2008JD009785, 2008.

Hatsushika, H. and Yamazaki, K.: Stratospheric drain over Indonesia and dehydration within the tropical tropopause layer diagnosed by air parcel trajectories, J. Geophys. Res., 108, 4610, doi:10.1029/2002JD002986, 2003.

Hitchman, M. H. and Leovy, C. B.: Estimation of the Kelvin wave contribution to the semiannual oscillation, J. Atmos. Sci., 45, 1462-1475, 1988.

Holton, J. R. and Lindzen, R. S.: An updated theory for the quasibiennial cycle of the tropical stratosphere, J. Atmos. Sci., 29, 1076-1080, 1972.

Immler, F., Krüger, K., Fujiwara, M., Verver, G., Rex, M., and Schrems, O.: Correlation between equatorial Kelvin waves and the occurrence of extremely thin ice clouds at the tropical tropopause, Atmos. Chem. Phys., 8, 4019-4026, 2008, 
http://www.atmos-chem-phys.net/8/4019/2008/.

Jensen, E. and Pfister, L.: Transport and freeze-drying in the tropical tropopause layer, J. Geophys. Res., 109, D02207, doi:10.1029/2003JD004022, 2004.

Jung, T. and Leutbecher, M.: Performance of the ECMWF forecasting system in the Arctic during winter, Q. J. R. Meteorol. Soc., 133, 1327-1340, doi:10.1002/qj.99, 2007.

Kawatani, Y., Takahashi, M., Sato, K., Alexander, S. P., and Tsuda, T.: Global distribution of atmospheric waves in the equatorial upper troposphere and lower stratosphere: AGCM simulation of sources and propagation, J. Geophys. Res., 114, D01102, doi:10.1029/2008JD010374, 2009.

Li, J., Wolf, W. W., Menzel, W. P., Zhang, W., Huang, H.-L., and Achtor, T. H.: Global soundings of the atmosphere from ATOVS measurements: The algorithm and validation, J. Appl. Meteorol., 39, 1248-1268, 2000.

Lindzen, R. S. and Holton, J. R.: A theory of the quasi-biennial oscillation, J. Atmos. Sci., 25, 1095-1107, 1968.

Lindzen, R. S.: The interaction of waves and convection in the tropics, J. Atmos. Sci., 60, 3009-3020, 2003.

Maruyama, T.: Upward transport of westerly momentum due to disturbances of the equatorial lower stratosphere in the period range of about 2 days - a Singapore data analysis for 1983-1993, J. Meteorol. Soc. Jpn., 72, 423-432, 1994.

Matsuno, T.: Quasi-geostrophic motions in the equatorial area, J. Meteorol. Soc. Jpn., 44, 25-43, 1966.

Mlynczak, M. G.: Energetics of the mesosphere and lower thermosphere and the SABER instrument, Adv. Space Res., 44, 11771183, 1997.

Mote, P. W., Rosenlof, K. H., Holton, J. R., Harwood, R. S., and Waters, J. W.: Seasonal variations of water vapor in the tropical lower stratosphere, Geophys. Res. Lett., 22, 1093-1096, 1995.

Mote, P. W., Rosenlof, K. H., McIntyre, M. E., Carr, E. S., Gille, J. C., Holton, J. R., Kinnersley, J. S., Pumphrey, H. C., Russell III, J. M., and Waters, J. W.: An atmospheric tape recorder: The imprint of tropical tropopause temperatures on stratospheric water vapor, J. Geophys. Res., 101, 3989-4006, 1996.

Preusse, P., Schroeder, S., Hoffmann, L., Ern, M., Friedl-Vallon, F., Oelhaf, H., Fischer, H., and Riese, M.: New perspectives on gravity wave remote sensing by spaceborne infrared limb imaging, Atmos. Meas. Tech. Discuss., 2, 825-856, 2009,

http://www.atmos-meas-tech-discuss.net/2/825/2009/.

Randel, W. J. and Wu, F.: Kelvin wave variability near the equatorial tropopause observed in GPS radio occultation measurements, J. Geophys. Res., 110, D03102, doi:10.1029/2004JD005006, 2005.

Ray, E. A., Alexander, M. J., and Holton, J. R.: An analysis of the structure and forcing of the equatorial semiannual oscillation in zonal wind, J. Geophys. Res., 103, 1759-1774, 1998.

Remsberg, E. E., Gordley, L. L., Marshall, B. T., Thompson, R. E., Burton, J., Bhatt, P., Harvey, V. L., Lingenfelser, G., and Natarajan, M.: The Nimbus 7 LIMS version 6 radiance conditioning and temperature retrieval methods and results, J. Quant. Spectrosc. Radiat. Transfer, 86, 395-424, doi:10.1016/j.jqsrt.2003.12.007, 2004.

Remsberg, E. E., Marshall, B. T., Garcia-Comas, M., Krueger, D., Lingenfelser, G. S., Martin-Torres, J., Mlynczak, M. G., Russell III, J. M., Smith, A. K., Zhao, Y., Brown, C., Gordley, L. L., Lopez-Gonzalez, M. J., Lopez-Puertas, M., She, C.-Y., Tay- lor, M. J., and Thompson, R. E.: Assessment of the quality of the Version 1.07 temperature-versus-pressure profiles of the middle atmosphere from TIMED/SABER, J. Geophys. Res., 113, D17101, doi:10.1029/2008JD010013, 2008.

Ricciardulli, L. and Garcia, R. R.: The excitation of equatorial waves by deep convection in the NCAR Community Climate Model (CCM3), J. Atmos. Sci., 57, 3461-3487, 2000.

Riese, M., Friedl-Vallon, F., Spang, R., Preusse, P., Schiller, C., Hoffmann, L., Konopka, P., Oelhaf, H., von Clarmann, T., and Höpfner, M.: GLObal limb Radiance Imager for the Atmosphere (GLORIA): Scientific objectives, Adv. Space Res., 36, 989-995, doi:10.1016/j.asr.2005.04.115, 2005.

Rosenlof, K.: Seasonal cycle of the residual mean circulation in the stratosphere, J. Geophys. Res., 100, 5173-5191, 1995.

Russell III, J. M., Mlynczak, M. G., Gordley, L. L., Tansock, J., and Esplin, R.: An overview of the SABER experiment and preliminary calibration results, Proceedings of SPIE, 3756, 277-288, 1999.

Salby, M. L.: Sampling theory for asynoptic satellite observations, Part I: Space-time spectra, resolution, and aliasing, J. Atmos. Sci., 39, 2577-2600, 1982a.

Salby, M. L.: Sampling theory for asynoptic satellite observations, Part II: Fast Fourier synoptic mapping, J. Atmos. Sci., 39, 26012614, 1982b.

Salby, M. L. and Garcia, R. R.: Transient response to localized episodic heating in the tropics, Part I: Excitation and short-time near-field behavior, J. Atmos. Sci., 44, 458-498, 1987.

Sato, K. and Dunkerton, T. J.: Estimates of momentum flux associated with equatorial Kelvin and gravity waves, J. Geophys. Res., 102(D22), 26247-26261, 1997.

Scaife, A., Butchart, N., Warner, C. D., Stainforth, D., and Norton, W.: Realistic quasi-biennial oscillations in a simulation of the global climate, Geophys. Res. Lett., 27, 3481-3484, 2000.

Schoeberl, M. R., Douglass, A. R., Stolarski, R. S., Pawson, S., Strahan, S. E., and Read, W.: Comparison of lower stratospheric tropical mean vertical velocities, J. Geophys. Res., 113, D24109, doi:10.1029/2008JD010221, 2008.

Schroeder, S., Preusse, P., Ern, M., and Riese, M.: Gravity waves resolved in ECMWF and measured by SABER, Geophys. Res. Lett., 36, L10805, doi:10.1029/2008GL037054, 2009.

Semeniuk, K. and Shepherd, T. G.: Mechanisms for tropical upwelling in the stratosphere, J. Atmos. Sci., 58, 3097-3115, 2001.

Shiotani, M. and Horinouchi, T.: Kelvin wave activity and the quasi-biennial oscillation in the equatorial lower stratosphere, J. Meteorol. Soc. Jpn., 71, 175-182, 1993.

Shuckburgh, E., Norton, W., Iwi, A., and Haynes, P.: Influence of the quasi-biennial oscillation on isentropic transport and mixing in the tropics and subtropics, J. Geophys. Res., 106(D13), 14327-14337, 2001.

Takahashi, M.: Simulation of the stratospheric quasi-biennial oscillation in a general circulation model, Geophys. Res. Lett., 26 1307-1310, 1999.

Tindall, J. C., Thuburn, J., and Highwood, E. J.: Equatorial waves in the lower stratosphere. I: A novel detection method, Q. J. R. Meteorol. Soc., 132, 177-194, doi:10.1256/qj.04.152, 2006a.

Tindall, J. C., Thuburn, J., and Highwood, E. J.: Equatorial waves in the lower stratosphere. II: Annual and interannual variability, Q. J. R. Meteorol. Soc., 132, 195-212, doi:10.1256/qj.04.153, 2006b. 
Uppala, S., Kallberg, P. W., Simmons, A. J., Andrae, U., Da Costa Bechtold, V., Fiorino, M., Gibson, J. K., Haseler, J., Hernandez, A., Kelly, G. A., Li, X., Onogi, K., Saarinen, S., Sokka, N., Allan, R. P., Andersson, E., Arpe, K., Balmaseda, M. A., Beljaars, A. C. M., van de Berg, L., Bidlot, J., Bormann, N., Caires, S., Chevallier, F., Dethof, A., Dragosavac, M., Fisher, M., Fuentes, M., Hagemann, S., Holm, E., Hoskins, B. J., Isaksen, L., Janssen, P. A. E. M., Jenne, R., McNally, A. P., Mahfouf, J.-F., Morcrette, J.-J., Rayner, N. A., Saunders, R. W., Simon, P., Sterl, A., Trenberth, K. E., Untch, A., Vasiljevic, D., Viterbo, P., and Woollen, J.: The ERA-40 re-analysis, Q. J. R. Meteorol. Soc., 131, 27612779, doi:10.1002/qj.99, 2005.

Venkat Ratnam, M., Tsuda, T., Kozu, T., and Mori, S.: Long-term behavior of the Kelvin waves revealed by CHAMP/GPS RO measurements and their effects on the tropopause structure, Ann. Geophys., 24, 1355-1366, 2006, http://www.ann-geophys.net/24/1355/2006/.

Wallace, M. W. and Kousky, V. E.: Observational evidence of Kelvin waves in the tropical stratosphere, J. Atmos. Sci., 25, 900-907, 1968.

Watanabe, S., Kawatani, Y., Tomikawa, Y., Miyazaki, K., Takahashi, M., and Sato, K.: General aspects of a T213L256 middle atmosphere general circulation model, J. Geophys. Res., 113, D12110, doi:10.1029/2008JD010026, 2008.
Wheeler, M. and Kiladis, G. N.: Convectively coupled equatorial waves: Analysis of clouds and temperature in the wavenumberfrequency domain, J. Atmos. Sci., 56, 374-399, 1999.

Wu, D. L., Hays, P. B., and Skinner, W. R.: A least squares method for spectral analysis of space-time series, J. Atmos. Sci., 52, 3501-3511, 1995.

Wu, Z., Sarachik, E. S., and Battisti, D. S.: Vertical structure of convective heating and the three-dimensional structure of the forced circulation on an equatorial beta plane, J. Atmos. Sci., 57, 21692187, 2000.

Yanai, M. and Maruyama, T.: Stratospheric wave disturbances propagating over the equatorial Pacific, J. Meteorol. Soc. Jpn., 44, 291-294, 1966.

Yee, J. H., Talaat, E. R., Christensen, A. B., Killeen, T. L., Russell, J. M., and Woods, T. N.: TIMED instruments, Johns Hopkins APL Technical Digest, 24, 156-164, 2003.

Zhou, X. and Holton, J. R.: Intraseasonal variations of tropical coldpoint tropopause temperatures, J. Climate, 15, 1460-1473, 2002. 